\title{
NECESSARY OPTIMALITY CONDITIONS FOR MINIMAX OPTIMAL CONTROL PROBLEMS WITH MIXED CONSTRAINTS
}

\author{
Paola Geovanna Patzi Aquino ${ }^{1}$, M.D.R. De Pinho ${ }^{2}$ \\ And Geraldo Nunes Silva ${ }^{3, *}$ (1)
}

\begin{abstract}
A weak maximal principle for minimax optimal control problems with mixed state-control equality and inequality constraints is provided. In the formulation of the minimax control problem we allow for parameter uncertainties in all functions involved: in the cost function, in the dynamical control system and in the equality and inequality constraints. Then a new constraint qualification of Mangassarian-Fromovitz type is introduced which allowed us to prove the necessary conditions of optimality. We also derived the optimality conditions under a full rank conditions type and showed that it is, as usual, a particular case of the Mangassarian-Fromovitz type condition case. Illustrative examples are presented.
\end{abstract}

Mathematics Subject Classification. 49J35, 49J15, 49J21.

Received July 16, 2020. Accepted June 22, 2021.

\section{INTRODUCTION}

Consider the following minimax optimal control problem with mixed constraints

$$
(P R) \quad\left\{\begin{array}{l}
\text { Minimize } \max _{\alpha \in \mathcal{A}} g(x(T, \alpha), \alpha) \\
\text { s.t } u:[S, T] \rightarrow \mathbb{R}^{k_{u}}, v:[S, T] \rightarrow \mathbb{R}^{k_{v}} \text { such that } v(t) \in V(t) \text { a.e. } t \in[S, T] \\
\text { and } \operatorname{arcs}\left\{x(\cdot, \alpha):[S, T] \rightarrow \mathbb{R}^{n} \mid \alpha \in \mathcal{A}\right\} \quad \text { such that, for each } \alpha \in \mathcal{A} \\
\dot{x}(t ; \alpha)=f(t, x(t, \alpha), u(t), v(t), \alpha), \quad \text { a.e. } t \in[S, T] \\
0=b(t, x(t, \alpha), u(t), v(t), \alpha) \\
0 \geq l(t, x(t, \alpha), u(t), v(t), \alpha) \\
x(S, \alpha)=x_{0} \\
x(T, \alpha) \in C(\alpha) .
\end{array}\right.
$$

Here $(\mathcal{A}, \rho)$ is an arbitrary compact metric space, $g: \mathbb{R}^{n} \times \mathcal{A} \rightarrow \mathbb{R},(f, b, l):[S, T] \times \mathbb{R}^{n} \times \mathbb{R}^{k_{u}} \times \mathbb{R}^{k_{v}} \times \mathcal{A} \rightarrow$ $\mathbb{R}^{n} \times \mathbb{R}^{m_{b}} \times \mathbb{R}^{m_{l}}$, are given functions, $V(t) \subset \mathbb{R}^{k_{v}}$ for all $t \in[S, T]$ is a time-dependent set, $C(\alpha) \subset \mathbb{R}^{n}$ is a

Keywords and phrases: Minimax optimal control problems; mixed constrained; maximum principle; nonsmooth analysis.

1 Universidad Mayor de San Andrés, Carrera de Matemática, La Paz, Bolivia.

${ }^{2}$ Universidade do Porto, Faculdade de Engenharia, DEEC, Systec, Porto, Portugal.

3 Departamento de Matemática, Instituto de Biociências, Letras e Ciências Exatas, UNESP - Universidade Estadual Paulista, São José do Rio Preto, SP, Brazil.

* Corresponding author; geraldo.silva@unesp.br 
closed set and $x_{0} \in \mathbb{R}^{n}$, with $m:=m_{b}+m_{l}, k:=k_{u}+k_{v}$ and $k \geq m$. We consider that the control variable is composed of two components $u$ and $v$. While $v(t)$ is allowed to take any value in a set $V(t)$, the component $u(t)$ is assumed to be free (unrestricted).

The optimization of dynamic systems, where the evaluation criterion is the maximum value of a function, is a problem of frequent occurrence in technology, economy and industry. This problem appears, in particular, when it is desired to minimize the maximum deviation of controlled trajectories in relation to a given model trajectory. Minimax-type problems differ from those usually considered in the optimal control literature, where a cumulative cost is minimized. Note that the minimax formulation of $(P R)$ takes into account the uncertainties of the parameters in both the cost function and the constraints of the control system. Minimization is taken over the worst case scenario, considering all the possible parameters, that is, we minimize over the feasible controls the maximum of a function over a set of parameters.

The new feature of $(P R)$ is the inclusion of the equality and inequality constraints depending on both the control and the state. An unrestricted version of this problem was recently addressed in the literature in [19], where R.B. Vinter obtained necessary conditions of optimality in the form of a Maximum Principle. These results were extended by Karamzin et al. in [10], where they provided necessary conditions of optimality for optimal minimax control problems with state constraints. Here we provide a further advance in the theory by furnishing necessary optimality conditions for minimax problems in the presence of mixed constraints.

In the case where $\mathcal{A}$ is a singleton, the mixed constrained minimax problem (PR) becomes a standard optimal control problem with mixed constraints, which has been the focus of attention for many years (see, for example $[2-5,7,9,13-17,20,21])$.

To obtain meaningful necessary conditions of optimality for these problems $(P R)$ one needs to impose some form of regularity condition or qualification on the mixed constraints. A simple one is the full rank of a certain matrix $F(t)$ in the sense that

$$
\operatorname{det} F(t) F(t)^{\top} \neq 0 \quad \text { for all } t \in[S, T]
$$

if $F(\cdot)$ is continuous. On the other hand, if the data of the problem are assumed to be merely measurable with respect a $t$, the full rank condition (1.1) is replaced by

$$
\operatorname{det} F(t) F(t)^{\top} \geq L \text { for almost all } t \in[S, T] \text { and for some } L>0 \text {. }
$$

For example, on $[9,14]$, the full rank condition (1.2) is imposed in the matrix

$$
\Upsilon_{1}(t)=\left(\begin{array}{c}
\nabla_{u} b(t, \bar{x}(t), \bar{u}(t), \bar{v}(t)) \\
\nabla_{u} l(t, \bar{x}(t), \bar{u}(t), \bar{v}(t))
\end{array}\right) .
$$

On [15] the full rank condition (1.2) is imposed in the matrix

$$
\Upsilon_{2}(t)=\left(\begin{array}{c}
\nabla_{u} b(t, \bar{x}(t), \bar{u}(t), \bar{v}(t)) \\
\nabla_{u} l^{\mathcal{I}_{\beta}(t)}(t, \bar{x}(t), \bar{u}(t), \bar{v}(t))
\end{array}\right)
$$

where $\mathcal{I}_{\beta}(t)=\left\{i \in\left\{1, \ldots, m_{l}\right\} \mid l_{i}(t, \bar{x}(t), \bar{u}(t), \bar{v}(t)) \geq-\beta\right\}$ and $\nabla_{u} l^{\mathcal{I}_{\beta}(t)}(t, \bar{x}(t), \bar{u}(t), \bar{v}(t))$ denotes the array we get after removing all index lines $i \notin \mathcal{I}_{\beta}(t)$ from $\nabla_{u} l(t, \bar{x}(t), \bar{u}(t), \bar{v}(t)), \beta>0$. In [16] and [3], (1.2) is imposed on

$$
\Upsilon_{3}(t)=\left(\begin{array}{cc}
\nabla_{u} b(t, \bar{x}(t), \bar{u}(t), \bar{v}(t)) & 0 \\
\nabla_{u} l(t, \bar{x}(t), \bar{u}(t), \bar{v}(t)) & \operatorname{diag}\left\{-l_{i}(t, \bar{x}(t), \bar{u}(t), \bar{v}(t))\right\}_{i \in\left\{1, \ldots, m_{l}\right\}}
\end{array}\right) .
$$


In Lemma 2.10.3 of [3] full rankness conditions imposed on the matrices above are related to each other and it is shown that the class of control problems in which

$$
F(t)=\left(\begin{array}{c}
\nabla_{u} b(t, \bar{x}(t), \bar{u}(t), \bar{v}(t)) \\
\nabla_{u} l^{\mathcal{I}_{a}(t)}(t, \bar{x}(t), \bar{u}(t), \bar{v}(t))
\end{array}\right)
$$

where $\mathcal{I}_{a}(t)=\left\{i \in\left\{1, \ldots, m_{l}\right\} \mid l_{i}(t, \bar{x}(t), \bar{u}(t), \bar{v}(t))=0\right\}$, satisfies full rankness condition is larger than the class of problems that satisfy the full rankness conditions (1.3), (1.4) and (1.5).

Another approach to get optimality conditions, more general than the full rankness condition (1.6), is based on the Mangasarian-Fromovitz type conditions which can be found in [4]. In [6] the case where equality and inequality constraints are not smooth is studied. We also refer the reader to the work of Li and Ye in $[11,12]$ for constant rank regularity conditions for autonomous problems.

Here we set out to obtain necessary optimality conditions for the minimax problem (PR) with mixed constraints, in which the set of parameters $\mathcal{A}$ is an arbitrary metric space. To do so, we introduce a new type of constraint qualifications generalizing the Mangasarian-Fromovitz conditions (MFC) tailored to derive the necessary conditions of optimality for the general minimax problem (PR). We allow for nonsmooth data and express necessary conditions in terms of limiting subdifferential and other nonsmooth analysis constructs. We obtain necessary conditions of optimality under MFC and show how to generalise the full rankness condition (FRC) to encompass minimax control problems with mixed constraints. Additionally, we discuss how FRC can be deduced as a particular case of the MFC and we also present an example illustrating that in the class of minimax control problems there are problems that satisfiy MFC and not FRC.

Here we treat minimax problems following the approach of [19]. Reformulation of minimax problems into bi-level problems in the spirit of [22] may be an alternative approach to ours. A discussion on such alternative would be long and quite technical. So we refrain to do it in this paper. This will howver be the focus of future work.

The paper is organized as follows: Section 2 presents some definitions and results on multifunction, differential inclusions and non-smooth analysis that will be used in the development of the work. In Section 3.2 we introduce regularity hypotheses into the constraints of the problem (PR), namely, two conditions of full-rank constraints and a Mangasarian-Fromovitz constraint condition. In the first part of Section 4 we provide necessary conditions for the problem (PR) when the set $\mathcal{A}$ is finite. Then we present a minimax example where the set $\mathcal{A}$ is an interval and we show that the obtained Maximum Principle ceases to be valid when $\mathcal{A}$ is an infinite set, making explicit the need to obtain new necessary optimality conditions when $\mathcal{A}$ is an arbitrary metric space. Yet in the same section we state the main result of the work, the necessary conditions for constrained minimax control problems, when $\mathcal{A}$ is an arbitrary metric space, as Theorem 4.4. Its proof is deferred to the Section 5.

\section{Preliminaries}

In this section we gather some basic notions and preliminary results that will be needed in this work. Here $|\cdot|$ denotes the Euclidean norm, $\mathbb{B}$ represents the closed unit ball centered at the origin, $\mathcal{L}$ denotes the Lebesgue subsets of $[S, T]$ and $\mathcal{B}^{p}$ and $\mathcal{B}^{\mathcal{A}}$ are taken to be the Borel subsets of $\mathbb{R}^{p}$ and $\mathcal{A}$, respectively. The vector spaces $L^{1}\left([S, T] ; \mathbb{R}^{p}\right), L^{\infty}\left([S, T] ; \mathbb{R}^{p}\right)$ and $W^{1,1}\left([S, T] ; \mathbb{R}^{p}\right)$ are the spaces of integrable functions, essentially bounded functions and absolutely continuous functions from $[S, T]$ to $\mathbb{R}^{p}$, respectively. $C(X)$ denotes the space of continuous real valued functions on $X$ and $C^{*}(X)$ the topological dual of $C(X)$. We make use of the well known fact that the space $C^{*}(X)$ can be identified with the space of Radon measures on Borel subsets of $X$. For more details, see [8].

A multifunction $D: X \subset \mathbb{R}^{n} \rightsquigarrow \mathbb{R}^{k}$ is a mapping from $X$ to the subsets of $\mathbb{R}^{k}$. A multifunction $D$ is called convex, closed, bounded or compact if for all $x \in X, D(x)$ has the property in question. The graph of a multifunction $D: X \rightsquigarrow \mathbb{R}^{k}$ is denoted by Gr $D$ and given by

$$
\operatorname{Gr} D:=\left\{(x, y) \in X \times \mathbb{R}^{k} \mid y \in D(x)\right\} .
$$


Given a closed set $C \subset \mathbb{R}^{k}$ and a point $x \in C$, the proximal normal cone to $C$ at $x$, written $N_{C}^{P}(x)$, is the set

$$
N_{C}^{P}(x):=\left\{p \in \mathbb{R}^{k} \mid \exists M>0 \text { such that } p \cdot(y-x) \leq M|y-x|^{2}, \quad \forall y \in C\right\}
$$

The limiting normal cone to $C$ at $x$, written $N_{C}(x)$, is the set

$$
N_{C}(x):=\left\{p \in \mathbb{R}^{k} \mid \text { there exists } x_{i} \stackrel{C}{\rightarrow} x, p_{i} \rightarrow p \text { such that } p_{i} \in N_{C}^{P}\left(x_{i}\right) \text { for all } i\right\} .
$$

Take a function $f: \mathbb{R}^{n} \rightarrow \mathbb{R} \cup\{+\infty\}$ and a point $x \in \operatorname{dom} f:=\left\{y \in \mathbb{R}^{n} \mid f(y)<+\infty\right\}$. The epigraph of $f$ is the set epi $f:=\left\{(x, y) \in \mathbb{R}^{n} \times \mathbb{R} \mid f(x) \leq y\right\}$. The limiting subdifferential $\partial f(x)$ of $f: \mathbb{R}^{n} \rightarrow \mathbb{R} \cup\{+\infty\}$ at a point $x \in \operatorname{dom} f$ is the set

$$
\partial f(x):=\left\{\eta \mid(\eta,-1) \in N_{\text {epi } f}(x, f(x))\right\} .
$$

Given $\delta>0$, the "truncation" $\operatorname{tr}_{\delta}: \mathbb{R}^{n} \rightarrow \mathbb{R}^{n}$, is defined by

$$
\operatorname{tr}_{\delta}(\xi):=\left\{\begin{array}{lll}
\xi, & \text { if } & |\xi| \leq \delta \\
\frac{\xi}{|\xi|} \delta, & \text { if } & |\xi|>\delta
\end{array}\right.
$$

Next, we state a result about measure convergence which we need in our analysis; for more details see Proposition 9.2.1 of [18] or Proposition 6.1 of [19].

Proposition 2.1. Take a compact metric space $X$, a sequence $\left\{\mu_{i}\right\}$ of nonnegative Radon measures in $C^{*}(X)$, a sequence $\left\{D_{i}: X \rightsquigarrow \mathbb{R}^{n}\right\}$ of multifunctions and a sequence of Borel measurable functions $\left\{\gamma_{i}: X \rightarrow \mathbb{R}^{n}\right\}$. Take also a measure $\mu \in C^{*}(X)$ and a multifunction $D: X \rightsquigarrow \mathbb{R}^{n}$. Assume that $\operatorname{Gr} D$ is compact, $D(x)$ is convex for each $x \in X, \limsup _{i \rightarrow \infty} \operatorname{Gr} D_{i} \subset \operatorname{Gr} D, \gamma_{i}(x) \in D_{i}(x) \mu_{i}$-a.e. $x \in X$ for $i=1,2, \ldots$ and $\mu_{i} \rightarrow \mu$ weakly ${ }^{*}$. Define $\eta_{i} \in C^{*}\left(X ; \mathbb{R}^{n}\right)$ according to $\eta_{i}(d x)=\gamma_{i}(x) \mu_{i}(d x) i=1,2, \ldots$ Then, along a subsequence, $\eta_{i} \rightarrow \eta$ weakly* for some $\eta \in C^{*}\left(X ; \mathbb{R}^{k}\right)$ and some Borel measurable function $\gamma$ such that

$$
\eta(d x)=\gamma(x) \mu(d x)
$$

and $\gamma(x) \in D(x)$, $\mu$-a.e.

Definition 2.2. A process $(u, v,\{x(\cdot, \alpha) \mid \alpha \in \mathcal{A}\})$ consists of a family of functions $\{x(\cdot, \alpha) \in$ $\left.W^{1,1}\left([S, T] ; \mathbb{R}^{n}\right) \mid \alpha \in \mathcal{A}\right\}$ and a pair of measurable functions $u:[S, T] \rightarrow \mathbb{R}^{k_{u}}$ and $v:[S, T] \rightarrow \mathbb{R}^{k_{v}}$, satisfying the constraints of the problem $(\mathrm{PR})$.

A process $(\bar{u}, \bar{v},\{\bar{x}(\cdot, \alpha) \mid \alpha \in \mathcal{A}\})$ is a weak minimizer if for some $\epsilon>0$

$$
\max _{\alpha \in \mathcal{A}} g(x(T, \alpha), \alpha) \geq \max _{\alpha \in \mathcal{A}} g(\bar{x}(T, \alpha), \alpha)
$$

for all process $(u, v,\{x(\cdot ; \alpha) \mid \alpha \in \mathcal{A}\})$ satisfying

$$
|x(t, \alpha)-\bar{x}(t, \alpha)| \leq \epsilon, \quad \text { for all }(t, \alpha) \in[S, T] \times \mathcal{A}
$$

and

$$
|u(t)-\bar{u}(t)| \leq \epsilon, \quad|v(t)-\bar{v}(t)| \leq \epsilon \text { a.e. } t \in[S, T] .
$$


For each $\alpha \in \mathcal{A}$, the set $\mathcal{I}_{a}^{\alpha}(t)$ denotes the set of indexes of the active constraints, that is,

$$
\mathcal{I}_{a}^{\alpha}(t)=\left\{i \in\left\{1, \ldots, m_{l}\right\} \mid l_{i}(t, \bar{x}(t, \alpha), \bar{u}(t), \bar{v}(t), \alpha)=0\right\},
$$

and $q_{a}^{\alpha}(t)$ denotes the cardinality of $\mathcal{I}_{a}^{\alpha}(t)$. In addition, for each $\alpha \in \mathcal{A}$, we have

$$
\nabla_{u} l^{\mathcal{I}_{a}^{\alpha}(t)}(t, \bar{x}(t, \alpha), \bar{u}(t), \bar{v}(t), \alpha) \in \mathbb{R}^{q_{a}^{\alpha}(t) \times k_{u}},
$$

which is the Jacobian matrix that we obtain from $\nabla_{u} l(t, \bar{x}(t, \alpha), \bar{u}(t), \bar{v}(t), \alpha)$ after removing all the lines of indexes $i \notin \mathcal{I}_{a}^{\alpha}(t)$.

\section{BASIC HYPOTHESES AND AUXILIARY RESUlts}

In this section we put together the basic assumptions and some auxiliary results that will be need for the main results.

\subsection{Hypotheses}

We pose the following hypotheses on (PR), which make reference to a scalar $\epsilon>0$ and a process $(\bar{u}, \bar{v},\{\bar{x}(\cdot ; \alpha) \mid \alpha \in \mathcal{A}\})$. The notation $(b, l)(\cdot)$ means $(b(\cdot), l(\cdot))$ and $\bar{b}(t, \alpha)=b(t, \bar{x}(t, \alpha), \bar{u}(t), \bar{v}(t), \alpha)$. For any $\alpha \in \mathcal{A}$ set

$$
\Omega_{\epsilon}(t, \alpha):=B(\bar{x}(t, \alpha), \epsilon) \times B(\bar{u}(t), \epsilon) \times(B(\bar{v}(t), \epsilon) \cap V(t)) .
$$

Let $\mathcal{W}:=\left\{(u, v):[S, T] \rightarrow \mathbb{R}^{k_{u}} \times \mathbb{R}^{k_{v}}:(u, v)(t)=(u(t), v(t)) \in(B(\bar{u}(t), \epsilon) \times B(\bar{v}(t), \epsilon)) \cap V(t)\right\}$ and $\Delta:$ $\mathcal{W} \times \mathcal{W} \rightarrow \mathbb{R}$ be the metric on $\mathcal{W}$ defined as

$$
\Delta\left((u, v),\left(u^{\prime}, v^{\prime}\right)\right)=\int_{S}^{T}\left(\left|u(t)-u^{\prime}(t)\right|+\left|v(t)-v^{\prime}(t)\right|\right) \mathrm{d} t .
$$

S1) The function $f(\cdot, x, u, v, \cdot)$ is $\mathcal{L} \times \mathcal{B}^{\mathcal{A}}$ measurable for each $(x, u, v)$ and there exists an integrable function $k_{f}$ and $C_{f}>0$ such that

$$
\left|f(t, x, u, v, \alpha)-f\left(t, x^{\prime}, u^{\prime}, v^{\prime}, \alpha\right)\right| \leq k_{f}\left|(x, u, v)-\left(x^{\prime}, u^{\prime}, v^{\prime}\right)\right| \quad \text { and } \quad|f(t, x, u, v, \alpha)| \leq C_{f},
$$

for all $(x, u, v),\left(x^{\prime}, u^{\prime}, v^{\prime}\right) \in \Omega_{\epsilon}(t, \alpha), \alpha \in \mathcal{A}$ and for almost every $t \in[S, T]$.

S2) The graph of $V$ is Borel measurable and $V_{\epsilon}(t)=B(\bar{v}(t), \epsilon) \cap V(t)$ is closed for almost every $t \in T$.

S3) $g(\cdot, \alpha)$ is locally Lipschitz on $\bar{x}(T, \alpha)+\epsilon B$, for all $\alpha \in \mathcal{A}$.

S4) $(b, l)(\cdot, x, u, v, \cdot)$ is $\mathcal{L} \times \mathcal{B}^{\mathcal{A}}$ measurable for each $(x, u, v)$, and the application $(t, \alpha) \mapsto l(t, \bar{x}(t, \alpha), \bar{u}(t), \bar{v}(t), \alpha)$ is $L^{\infty}\left([S, T] \times \mathcal{A}, \mathbb{R}^{m_{g}}\right)$.

S5) $(b, l)(t, \cdot, \cdot, \cdot, \alpha)$ is continuously differentiable on $(\bar{x}(t, \alpha), \bar{u}(t), \bar{v}(t))+\epsilon B$ a.e. $t \in[S, T]$ and there exists an integrable function $L_{b, l}$ such that, for almost every $t \in[S, T]$ and for each $\alpha \in \mathcal{A}$,

$$
\left|(b, l)(t, x, u, v, \alpha)-(b, l)\left(t, x^{\prime}, u^{\prime}, v^{\prime}, \alpha\right)\right| \leq L_{b, l}(t)\left|(x, u, v)-\left(x^{\prime}, u^{\prime}, v^{\prime}\right)\right|,
$$

for all $(x, u, v),\left(x^{\prime}, u^{\prime}, v^{\prime}\right) \in \Omega_{\epsilon}(t, \alpha)$.

S6) There exists $K_{b, l}>0$ and an increasing function $\theta:(0, \infty) \rightarrow(0, \infty)$, with $\lim _{s \downarrow 0} \theta(s)=0$, such that, for almost every $t \in[S, T]$ and for all $\alpha \in \mathcal{A}$,

$$
\left|\nabla_{x}(\bar{b}, \bar{l})(t, \alpha)\right|+\left|\nabla_{u}(\bar{b}, \bar{l})(t, \alpha)\right|+\left|\nabla_{v}(\bar{b}, \bar{l})(t, \alpha)\right| \leq K_{b, l},
$$


and

$$
\left|\nabla_{x, u, v}(b, l)(t, x, u, v, \alpha)-\nabla_{x, u, v}(b, l)\left(t, x^{\prime}, u^{\prime}, v^{\prime}, \alpha\right)\right| \leq \theta\left(\left|(x, u, v)-\left(x^{\prime}, u^{\prime}, v^{\prime}\right)\right|\right)
$$

for all $(x, u, v) \neq\left(x^{\prime}, u^{\prime}, v^{\prime}\right) \in \Omega_{\epsilon}(t, \alpha)$.

S7) The application $\alpha \mapsto(b, l)_{x, u, v}(t, x, u, v, \alpha)$ is uniformly continuous with respect to $(t, x, u, v) \in$ $\left\{\left(t, x^{\prime}, u^{\prime}, v^{\prime}\right) \in[S, T] \times \mathbb{R}^{n} \times \mathbb{R}^{k_{u}} \times \mathbb{R}^{k_{v}} \mid v^{\prime} \in V\left(t^{\prime}\right)\right\}$.

S8) There exists $\omega:(0, \infty) \rightarrow(0, \infty)$ such that $\lim _{s \downarrow 0} \omega(s)=0$ and, for all $\alpha, \alpha^{\prime} \in \mathcal{A}$,

$$
\int_{S}^{T} \sup _{(x, u, v) \in \Omega_{\epsilon}(t, \alpha)}\left|f(t, x, u, v, \alpha)-f\left(t, x, u, v, \alpha^{\prime}\right)\right| \mathrm{d} t \leq \omega\left(\rho\left(\alpha, \alpha^{\prime}\right)\right) .
$$

S9) $g(x, \cdot)$ and $\alpha \mapsto d_{C(\alpha)}(x)$ are continuous on $\mathcal{A}$ for each $x \in \mathbb{R}^{n}$.

In addition to the hypotheses S1)-S9) we need regularity conditions on the constraints of equality and inequality, called constraint qualifications. This is the subject of the next subsection.

\subsection{Constraints qualifications}

Here, we present the constraints qualifications for minimax control problems. We first introduce the Mangasarian-Fromovitz (MFC) type constraint qualifications and then the full rank type conditions.

\subsubsection{Mangasarian-Fromovitz type conditions}

The following hypothesis is said to be a Mangasarian-Fromovitz type condition because of similarities to the classical mathematical programming case.

(MFC) There exists $K_{1}>0$, a function $h \in L^{\infty}\left([S, T] ; \mathbb{R}^{k_{u}}\right)$ and a family of functions $\{a(\cdot, \alpha):[S, T] \rightarrow$ $\left.\mathbb{R}^{m_{l}} \mid \alpha \in \mathcal{A}\right\} \in L^{\infty}$ with $|h(t)|=1$ a.e. $t \in[S, T]$ such that, for almost every $t \in[S, T]$ and for all $\alpha \in \mathcal{A}$,

i) $a_{j}(t, \alpha)>k_{1}$ for $j \in \mathcal{I}_{a}^{\alpha}(t)$,

ii) $\nabla_{u} l^{\mathcal{I}_{a}^{\alpha}(t)}(t, \bar{x}(t, \alpha), \bar{u}(t), \bar{v}(t), \alpha) \cdot h(t)=a^{\mathcal{I}_{a}^{\alpha}(t)}(t, \alpha)$,

iii) $\nabla_{u} b(t, \bar{x}(t, \alpha), \bar{u}(t), \bar{v}(t), \alpha) \cdot h(t)=0$,

iv) There exist $M>0$ such that for any finite subset $\widetilde{\mathcal{A}}=\left\{\alpha_{1}, \ldots, \alpha_{k}^{i}\right\}$ of $\mathcal{A}$

$$
\operatorname{det}\left(\begin{array}{cccc}
F^{\alpha_{1}}(t) F^{\alpha_{1}}(t)^{\top} & F^{\alpha_{1}}(t) F^{\alpha_{2}}(t)^{\top} & \cdots & F^{\alpha_{1}}(t) F^{\alpha_{k}^{i}}(t)^{\top} \\
F^{\alpha_{2}}(t) F^{\alpha_{1}}(t)^{\top} & F^{\alpha_{2}}(t) F^{\alpha_{2}}(t)^{\top} & \cdots & F^{\alpha_{2}}(t) F^{\alpha_{k}^{i}}(t)^{\top} \\
\vdots & \vdots & \ddots & \vdots \\
F^{\alpha_{k}^{i}}(t) F^{\alpha_{1}}(t)^{\top} & F^{\alpha_{k}^{i}}(t) F^{\alpha_{2}}(t)^{\top} & \cdots & F^{\alpha_{k}^{i}}(t) F^{\alpha_{k}^{i}}(t)^{\top}
\end{array}\right) \geq M \quad \text { a.e.t } \in[S, T]
$$

where $F^{\alpha}(t)=\nabla_{u} b(t, \bar{x}(t, \alpha), \bar{u}(t), \bar{v}(t), \alpha)$.

\subsubsection{Full rank type conditions}

Since the data of (PR) depend on parameters and are only measurable with respect to $t$, the condition (1.2) must be replaced by: A1) or A2).

A1) i) There exists $k>0$ such that $\operatorname{det}\left\{F_{M}^{\alpha}(t) F_{M}^{\alpha}(t)^{T}\right\} \geq k$ a.e. $t \in[S, T]$ and $\alpha \in \mathcal{A}$, where

$$
F_{M}^{\alpha}(t)=\left(\begin{array}{c}
\nabla_{u} b(t, \bar{x}(t, \alpha), \bar{u}(t), \bar{v}(t), \alpha) \\
\nabla_{u} l^{\mathcal{I}_{a}^{\alpha}(t)}(t, \bar{x}(t, \alpha), \bar{u}(t), \bar{v}(t), \alpha)
\end{array}\right)
$$


ii) If $\alpha \neq \alpha^{\prime}$ then $F_{M}^{\alpha}(t) F_{M}^{\alpha^{\prime}}(t)^{\top}=0$;

A2) There exists a constant $M>0$ such that for any finite subset $\widetilde{\mathcal{A}}=\left\{\alpha_{1}, \ldots, \alpha_{k}^{i}\right\}$ of $\mathcal{A}$ we have

$$
\operatorname{det}\left(\begin{array}{cccc}
F_{M}^{\alpha_{1}}(t) F_{M}^{\alpha_{1}}(t)^{\top} & F_{M}^{\alpha_{1}}(t) F_{M}^{\alpha_{2}}(t)^{\top} & \cdots & F_{M}^{\alpha_{1}}(t) F_{M}^{\alpha_{k}^{i}}(t)^{\top} \\
F_{M}^{\alpha_{2}}(t) F_{M}^{\alpha_{1}}(t)^{\top} & F_{M}^{\alpha_{2}}(t) F_{M}^{\alpha_{2}}(t)^{\top} & \cdots & F_{M}^{\alpha_{2}}(t) F_{M}^{\alpha_{k}^{i}}(t)^{\top} \\
\vdots & \vdots & \ddots & \vdots \\
F_{M}^{\alpha_{k}^{i}}(t) F_{M}^{\alpha_{1}}(t)^{\top} & F_{M}^{\alpha_{k}^{i}}(t) F_{M}^{\alpha_{2}}(t)^{\top} & \cdots & F_{M}^{\alpha_{k}^{i}}(t) F_{M}^{\alpha_{k}^{i}}(t)^{\top}
\end{array}\right) \geq M \quad \text { a.e. } t \in[S, T] .
$$

When $\mathcal{A}$ is a unitary set the conditions, A1) and A2) are equivalent.

It is easy to verify that the hypothesis (MFC) is weaker than the hypotheses A1) or A2). We shall see an example in Section 4 where the Mangasarian-Fromovitz condition is less restrictive than the full-rank conditions.

\subsection{Auxiliary results}

The following lemma gathers some useful facts regarding the dependence of the trajectories on the controls and parameters. To emphasise these dependences we use the notation $x(\cdot, \alpha, u, v)$.

Lemma 3.1. Assume that S1), S2) and S8) are valid. Then, for any $\delta>0$, a finite subset $\widetilde{\mathcal{A}} \subset \mathcal{A}$ and $\rho>0$ can be chosen such that:

i) $\sup _{(u, v) \in \mathcal{U} \times \mathcal{V}} \sup _{\alpha \in \mathcal{A}} \inf _{\alpha^{\prime} \in \widetilde{\mathcal{A}}}\left\|x(\cdot, \alpha, u, v)-x\left(\cdot, \alpha^{\prime}, u, v\right)\right\|<\delta$,

ii) $\sup _{\alpha \in \mathcal{A}}\left\{\left\|x(\cdot ; \alpha, u, v)-x\left(\cdot ; \alpha, u^{\prime}, v^{\prime}\right)\right\| \mid(u, v),\left(u^{\prime}, v^{\prime}\right) \in \mathcal{W}, \Delta\left((u, v),\left(u^{\prime}, v^{\prime}\right)\right)<\rho\right\}<\delta$.

Proof. Note that $i$ ) follows from

$$
\left|x(s, \alpha, u)-x\left(s, \alpha^{\prime}, u\right)\right| \leq \omega\left(\rho\left(\alpha, \alpha^{\prime}\right)\right) \exp \left(\int_{0}^{s} k_{f}(\tau) \mathrm{d} \tau\right)
$$

which is valid because of the compactness of $\mathcal{A}$ and the Gronwall's Inequality. By making use of the Dunfort Pettis Criterion we obtain $i$ ).

Let us define the Hamiltonian function as follows:

$$
H(t, x, p, q, r, u, v, \alpha)=p \cdot f(t, x, u, v, \alpha)+q \cdot b(t, x, u, v, \alpha)+r \cdot l(t, x, u, v, \alpha) .
$$

Lemma 3.2. If S1) and S5) are satisfied, then the Hamiltonian function $H(t, \cdot, p, q, r, \cdot, \cdot, \alpha)$ is Lipschitz continuous with respect to $(x, u, v) \in \mathbb{R}^{n} \times \mathbb{R}^{k_{u}} \times \mathbb{R}^{k_{v}}$, for all $(t, p, q, r) \in[S, T] \times \mathbb{R}^{n} \times \mathbb{R}^{m_{b}} \times \mathbb{R}^{m_{l}}$.

Proof. It follows immediately from the hypotheses S1) and S5).

Lemma 3.3. Let $f_{i}: \mathbb{R}^{n} \times \mathbb{R}^{k} \rightarrow \mathbb{R}, i=1,2, \cdots, N$, be given Lipschitz funcions and $f(x, u)=f_{1}\left(x_{1}, u\right)+$ $f_{2}\left(x_{2}, u\right)+\cdots+f_{N}\left(x_{N}, u\right)$, where $x=\left(x_{1}, x_{2}, \ldots, x_{N}\right)$ and $x_{i} \in \mathbb{R}^{n}$. Then

$$
(\xi, \gamma) \in \partial^{P} f(x, u) \Leftrightarrow \exists \eta_{i} \in \mathbb{R}^{k} \text { such that }\left(\xi_{i}, \eta_{i}\right) \in \partial^{P} f_{i}\left(x_{i}, u\right) \text { and } \gamma=\sum_{j=1}^{N} \eta_{j} \text {, with } \xi=\left(\xi_{1}, \xi_{2}, \ldots, \xi_{N}\right)
$$


Proof. Let

$$
F(x, u, y)=\sum_{j=1}^{N} f_{j}\left(x_{j}, u+y_{j}\right)
$$

where $x=\left(x_{1}, \ldots, x_{N}\right), x_{i} \in \mathbb{R}^{n}$, e $y=\left(y_{1}, \ldots, y_{N}\right), y_{i} \in \mathbb{R}^{k}$. Note that $F(x, u, 0)=\sum_{j=1}^{N} f_{j}\left(x_{j}, u\right)=f(x, u)$.

Let $(\xi, \gamma, \eta) \in \partial^{P} F(x, u, y)$. Then, there exist $M>0$ such that

$$
(\xi, \gamma, \eta) \cdot\left(\left(x^{\prime}, u^{\prime}, y^{\prime}\right)-(x, u, y)\right) \leq F\left(x^{\prime}, u^{\prime}, y^{\prime}\right)-F(x, u, y)+M\left|\left(x^{\prime}, u^{\prime}, y^{\prime}\right)-(x, u, y)\right|^{2},
$$

for all $\left(x^{\prime}, u^{\prime}, y^{\prime}\right) \in \mathbb{R}^{N \times n} \times \mathbb{R}^{k} \times \mathbb{R}^{N \times k}$, where $x^{\prime}=\left(x_{1}^{\prime}, \ldots, x_{N}^{\prime}\right)$ and $y^{\prime}=\left(y_{1}^{\prime}, \ldots, y_{N}^{\prime}\right)$.

Therefore, it follows that

$$
\begin{array}{r}
\gamma \cdot\left(u^{\prime}-u\right)+\sum_{j=1}^{N}\left[\xi_{j} \cdot\left(x_{j}^{\prime}-x_{j}\right)+\eta_{j} \cdot\left(y_{j}^{\prime}-y_{j}\right)\right] \leq \sum_{j=1}^{N}\left[f_{j}\left(x_{j}^{\prime}, u^{\prime}+y_{j}^{\prime}\right)-f_{j}\left(x_{j}, u+y_{j}\right)\right] \\
+M\left(\left|\left(u^{\prime}-u\right)\right|^{2}+\sum_{j=1}^{N}\left|\left(x_{j}^{\prime}, y_{j}^{\prime}\right)-\left(x_{j}, y_{j}\right)\right|^{2}\right),
\end{array}
$$

for all $\left(x^{\prime}, u^{\prime}, y^{\prime}\right)$. In particular, for $x^{\prime}=\left(x_{1}, \ldots, x_{i}^{\prime}, \ldots, x_{N}\right), u^{\prime}=u$ and $y^{\prime}=\left(y_{1}, \ldots, y_{i}^{\prime}, \ldots, y_{N}\right)$, we have

$$
\left(\xi_{i}, \eta_{i}\right) \cdot\left(\left(x_{i}^{\prime}, y_{i}^{\prime}\right)-\left(x_{i}, y_{i}\right)\right) \leq f_{i}\left(x_{i}^{\prime}, u+y_{i}^{\prime}\right)-f_{i}\left(x_{i}, u+y_{i}\right)+M\left|\left(x_{i}^{\prime}, y_{i}^{\prime}\right)-\left(x_{i}, y_{i}\right)\right|^{2}
$$

Thus, $\left(\xi_{i}, \eta_{i}\right) \in \partial^{P} f_{i}\left(x_{i}, u+y_{i}\right)$.

Take $u^{\prime}$ close to $u, x_{i}^{\prime}=x_{i}$ and $y_{i}^{\prime}=u+y_{i}-u^{\prime}$. It follows from (3.3) that

$$
\gamma \cdot\left(u^{\prime}-u\right)+\sum_{j=1}^{N}\left[\eta_{j} \cdot\left(u-u^{\prime}\right)\right] \leq M\left[\left|u^{\prime}-u\right|^{2}+\sum_{j=1}^{N}\left(\left|u^{\prime}-u\right|^{2}\right)\right] .
$$

Consequently

$$
\left(\gamma-\sum_{j=1}^{N} \eta_{j}\right) \cdot\left(u^{\prime}-u\right) \leq N^{\prime}\left|u^{\prime}-u\right|^{2}, \quad \text { where } N^{\prime}=M(1+N), \text { for all } u^{\prime} \in \mathbb{R}^{k},
$$

then $\left(\gamma-\sum_{j=1}^{N} \eta_{j}\right) \in N_{\mathbb{R}^{k}}^{P} u=\{0\}$, therefore $\gamma=\sum_{j=1}^{N} \eta_{j}$. Thereby,

$$
\partial^{P} F(x, u, y) \subset\left\{(\xi, \gamma, \eta):\left(\xi_{i}, \eta_{i}\right) \in \partial^{P} f_{i}\left(x_{i}, u+y_{i}\right) \text { and } \gamma=\sum_{i=1}^{N} \eta_{i}\right\} .
$$


The other inclusion is immediately, in fact, considering $\left(\xi_{i}, \eta_{i}\right) \in \partial^{P} f_{i}\left(x_{i}, u\right), \gamma=\sum_{i=1}^{N} \eta_{i}, \xi=\left(\xi_{i}, \ldots, \xi_{N}\right)$, the definition of proximal subdifferential and taking the respective summations, we obtain

$$
(\xi, \eta)\left(\left(x^{\prime}, u^{\prime}\right)-(x, u)\right) \leq \sum_{i} f_{i}\left(x_{i}^{\prime}, u^{\prime}\right)-\sum_{i} f_{i}\left(x_{i}, u\right)+M^{\prime}\left|\left(x^{\prime}, u^{\prime}\right)-(x, u)\right|
$$

Since $F(x, u, 0)=\sum_{j=1}^{N} f_{j}\left(x_{j}, u\right)=f(x, u)$, we have

$$
(\xi, \eta)\left(\left(x^{\prime}, u^{\prime}\right)-(x, u)\right) \leq f\left(x^{\prime}, u^{\prime}\right)-f(x, u)+M^{\prime}\left|\left(x^{\prime}, u^{\prime}\right)-(x, u)\right|,
$$

so $(\xi, \eta) \in \partial^{P} f(x, u)$. Therefore,

$$
\partial^{P} f(x, u)=\partial_{x, u} F(x, u, 0)=\left\{\left(\xi, \gamma=\sum_{i=1}^{N} \eta_{i}\right):\left(\xi_{i}, \eta_{i}\right) \in \partial^{P} f_{i}\left(x_{i}, u\right)\right\} .
$$

\section{Necessary optimality Conditions for the Problem (PR)}

In this section we state the main result of this work as Theorem 4.4, which gives necessary optimality conditions for the general minimax control problem (PR) with equality and inequality constraints and when the set $\mathcal{A}$ is an arbitrary metric space. The proof involves new mathematical constructs and ideas inspired by the works [19] and [10].

Roughly speaking, the proof is based on approximating the compact metric space $\mathcal{A}$ by a family of finite subsets $\left\{\mathcal{A}_{k}\right\}_{k=1}^{\infty}$ ordered in such way that the next member of the family contains the previous one. First we show, as Proposition 4.2 below, that when $\mathcal{A}$ is finite and any of the constraint qualifications A1), A2) or (MFC) is valid, the necessary optimality conditions hold true. Next we show that optimal solutions of the general minimax problem can be approximated by optimal solutions of the approximated problems with finite set of parameters. Since necessary conditions of optimality are available for these minimax problems as Propostion 4.2, we need to prove that these conditions are still valid when we make A infinite by taking limits. This is the main part of the proof.

As the proof of Theorem 4.4 is long and involved we leave it for a separate section. We now make some preparations for stating the main result later in this section.

\subsection{The case in which the set $\mathcal{A}$ is finite}

We observe that when $\mathcal{A}$ is a singleton, we have the following necessary optimality conditions in the form of the non-smooth Maximum Principle, stated as Proposition 4.1, which is an immediate consequence of Theorem 3.1 in [2].

Proposition 4.1. ([2], Thm. 3.1) Let $(\bar{x}, \bar{u}, \bar{v})$ be a weak minimizer for (PR). Assume that $\mathcal{A}$ is a singleton and that S1)-S6) and A1) (or A2)) are satisfied. Then there exist $p \in W^{1,1}\left([S, T] ; \mathbb{R}^{n}\right), q \in L^{1}\left([S, T] ; \mathbb{R}^{m_{b}}\right)$, $r \in L^{1}\left([S, T] ; \mathbb{R}^{m_{l}}\right), \xi \in L^{1}\left([S, T] ; \mathbb{R}^{k_{v}}\right)$ and $\lambda \geq 0$ such that:

i) $\|p\|_{\infty}+\lambda \neq 0$;

ii) $(-\dot{p}(t), 0, \xi(t)) \in \operatorname{co} \partial_{x, u, v} H(t, \bar{x}(t), p(t), q(t), r(t), \bar{u}(t), \bar{v}(t), \alpha)$, a.e. $t \in[S, T]$;

iii) $\xi(t) \in \operatorname{co} N_{V(t)}(\bar{v}(t))$;

iv) $r(t) \cdot l(t, \bar{x}(t), \bar{u}(t), \bar{v}(t), \alpha)=0$ and $r(t) \leq 0$ a.e. in $t \in[S, T]$; 
v) $-p(T) \in N_{S}(\bar{x}(T))+\lambda \partial g(\bar{x}(T))$.

In addition, for some integrable $K_{m}$,

$$
|(q(t), r(t))| \leq K_{m}(t)|p(t)| \quad \text { a.e. } \quad t \in[S, T] .
$$

In [4] it is shown that Proposition 4.1 is still valid when the hypotheses A1) or A2) are replaced by the Mangasarian-Fromovitz condition (MFC).

When $\mathcal{A}$ is finite, the problem (PR) can be rewritten as a minimization problem with equality and inequality constraints with respect to the state and control, for which we may apply existing necessary conditions in the form of a weak maximum principle found in [2] and [4], to obtain the following result.

Proposition 4.2. Let $(\bar{u}, \bar{v},\{\bar{x}(\cdot ; \alpha) \mid \alpha \in \mathcal{A}\})$ be a weak minimizer for (PR). Assume that S1) - S6) and A1) (or A2) or (MFC)) are satisfied and that $\mathcal{A}$ is a finite set. Then, there are families of multipliers $\{\lambda(\alpha) \in[0,1] \mid \alpha \in$ $\mathcal{A}\},\left\{p(\cdot, \alpha) \in W^{1,1}\left([S, T] ; \mathbb{R}^{n}\right) \mid \alpha \in \mathcal{A}\right\},\left\{q(\cdot, \alpha) \in L^{1}\left([S, T] ; \mathbb{R}^{m_{b}}\right)\right\},\left\{r(\cdot, \alpha) \in L^{1}\left([S, T] ; \mathbb{R}^{m_{l}}\right)\right\}, \quad\{\xi(\cdot, \alpha) \in$ $\left.L^{1}\left([S, T] ; \mathbb{R}^{k_{v}}\right)\right\}$ and probability measure $\Lambda$ such that, for $\Lambda$-a.e. $\alpha \in \mathcal{A}$,

i) $\|p(\cdot, \alpha)\|_{\infty}+\lambda(\alpha)=1$;

ii) $(\dot{p}(t, \alpha), 0, \xi(t, \alpha)) \in \operatorname{co} \partial_{x, u, v} H(t, \bar{x}(t, \alpha), p(t, \alpha), q(t, \alpha), r(\cdot, \alpha), \bar{u}(t), \bar{v}(t), \alpha) \quad$ a.e. $t \in[S, T]$;

iii) $\xi(t)=\int_{\mathcal{A}} \xi(t, \alpha) \mathrm{d} \Lambda(\alpha)$ and $\xi(t) \in \operatorname{co} N_{V(t)}(\bar{v}(t))$ a.e. $t \in[S, T]$;

iv) $-p(T, \alpha) \in \lambda(\alpha) \partial g(\bar{x}(T, \alpha), \alpha)+N_{C_{\alpha}}(\bar{x}(T, \alpha))$;

v) $\int_{\mathcal{A}} r(t, \alpha) \cdot l(t, \bar{x}(t, \alpha), \bar{u}(t), \bar{v}(t), \alpha) \mathrm{d} \Lambda(\alpha)=0 \quad$ and $\quad r(t, \alpha) \leq 0 \quad$ a.e. $t \in[S, T]$;

vi) Furthermore, for some integrable function $K_{m}$,

$$
\int_{\mathcal{A}}(|q(t, \alpha)|+|r(t, \alpha)|) \mathrm{d} \Lambda(\alpha) \leq K_{m}(t) \int_{\mathcal{A}}|p(t, \alpha)| \mathrm{d} \Lambda(\alpha) \text { a.e. } t \in[S, T] .
$$

Proof. Assume that $\mathcal{A}=\left\{\alpha_{1}, \alpha_{2}, \ldots, \alpha_{N}\right\}$. Here we use the state augmentation techniques. Let $\bar{x}=$ $\operatorname{col}\left\{\bar{x}\left(\cdot, \alpha_{1}\right), \bar{x}\left(\cdot, \alpha_{2}\right), \ldots, \bar{x}\left(\cdot, \alpha_{N}\right)\right\}$ be the collection of state trajectories corresponding to $(\bar{u}, \bar{v})$. Then $(\bar{u}, \bar{v}, \bar{x})$ is a minimizer for the optimal control problem $(\widetilde{P R})$, given by

$$
(\widetilde{P R}) \quad\left\{\begin{array}{l}
\text { Minimize } \tilde{g}(x(T)) \\
\dot{x}(t)=\tilde{f}(t, x(t), u(t), v(t)), \quad \text { a.e. } \quad t \in[S, T], \\
0=\tilde{b}(t, x(t), u(t), v(t)), \quad \text { a.e. } t \in[S, T], \\
0 \geq \tilde{l}(t, x(t), u(t), v(t)), \quad \text { a.e. } t \in[S, T], \\
v(t) \in V(t), \\
x(S)=\tilde{x}_{0}, \\
x(T) \in \widetilde{C},
\end{array}\right.
$$

where

$$
\begin{aligned}
x & =\operatorname{col}\left\{x_{1}, x_{2}, \ldots, x_{N}\right\}, x_{i} \in \mathbb{R}^{n}, i=1, \ldots, N, \\
\tilde{f}(t, x, u, v) & =\operatorname{col}\left\{f\left(t, x_{i}, u, v, \alpha_{i}\right)\right\}_{i=1}^{N}, \\
\tilde{b}(t, x, u, v) & =\operatorname{col}\left\{b\left(t, x_{i}, u, v, \alpha_{i}\right)\right\}_{i=1}^{N}, \\
\tilde{l}(t, x, u, v) & =\operatorname{col}\left\{l\left(t, x_{i}, u, v, \alpha_{i}\right)\right\}_{i=1}^{N}, \\
\tilde{x}_{0} & =\operatorname{col}\left\{x_{0}, x_{0}, \ldots, x_{0}\right\} \\
\tilde{g}(x(\cdot)) & =\max _{1 \leq i \leq N} g\left(x\left(\cdot, \alpha_{i}\right), \alpha_{i}\right),
\end{aligned}
$$


and $\widetilde{C}=C\left(\alpha_{1}\right) \times \cdots \times C\left(\alpha_{N}\right)$.

The problem $(\widetilde{P R})$ is a standard minimization problem with constraints, for which all hypotheses are satisfied. Applying Proposition 4.1 to $(\widetilde{P R})$ and appealing to the Maximum Rule ([18], Thm. 5.5.2) and Lemma 3.3, we deduce the existence of $p\left(\cdot, \alpha_{i}\right) \in W^{1,1}\left([S, T] ; \mathbb{R}^{n}\right), q\left(\cdot, \alpha_{i}\right) \in L^{1}\left([S, T] ; \mathbb{R}^{m_{b}}\right), r\left(\cdot, \alpha_{i}\right) \in L^{1}\left([S, T] ; \mathbb{R}^{m_{g}}\right), \xi\left(\cdot, \alpha_{i}\right) \in$ $L^{1}\left([S, T] ; \mathbb{R}^{k_{v}}\right)$ and $\lambda \geq 0$ such that

(a) $\sum_{i=1}^{N}\left\|p\left(\cdot, \alpha_{i}\right)\right\|_{\infty}+\lambda \neq 0$

(b) $\left(-\dot{p}\left(t, \alpha_{i}\right), 0, \xi(t, \alpha)\right) \in \operatorname{co} \partial_{x, u, v} H\left(t, \bar{x}\left(t, \alpha_{i}\right), p\left(t, \alpha_{i}\right), q\left(t, \alpha_{i}\right), r\left(t, \alpha_{i}\right), \bar{u}(t), \bar{v}(t), \alpha_{i}\right)$, for $i=1, \ldots, N$;

(c) $\xi=\sum_{i=1}^{N} \xi\left(\cdot, \alpha_{i}\right) \quad$ and $\quad \xi(t) \in \operatorname{co} N_{V(t)}(\bar{v}(t))$;

(d) $\sum_{i=1}^{N} r\left(t, \alpha_{i}\right) \cdot l\left(t, \bar{x}\left(t, \alpha_{i}\right), \bar{u}(t), \bar{v}(t), \alpha_{i}\right)=0$ and $r\left(t, \alpha_{i}\right) \leq 0$ a.e. $t \in[S, T]$;

(e) $-p\left(T, \alpha_{i}\right) \in N_{C\left(\alpha_{i}\right)}\left(\bar{x}\left(T, \alpha_{i}\right)\right)+\lambda \beta_{i} \partial g\left(\bar{x}\left(T, \alpha_{i}\right), \alpha_{i}\right)$, with $\beta_{i} \geq 0$ for $i=1, \ldots, N, \beta_{i}=0$ if $g\left(\bar{x}\left(T, \alpha_{i}\right), \alpha_{i}\right)<$ $\max _{j} g\left(\bar{x}\left(T, \alpha_{j}\right), \alpha_{j}\right)$ and $\sum_{i=1}^{N} \beta_{i}=1 ;$

(f) for some integrable function $K_{m}$,

$$
\sum_{i=1}^{N}\left|q\left(t, \alpha_{i}\right)\right|+\left|r\left(t, \alpha_{i}\right)\right| \leq K_{m}(t) \sum_{i=1}^{N}\left|p\left(t, \alpha_{i}\right)\right| .
$$

The nontriviality of the multiplers $(a)$ allows us to choose new multipliers satisfying $(b)-(f)$ and such that $(a)$ is replaced by

$$
\sum_{i=1}^{N}\left\|p\left(\cdot, \alpha_{i}\right)\right\|+\lambda=1
$$

Let $c_{i}:=\left\|p\left(\cdot, \alpha_{i}\right)\right\|_{\infty}+\lambda \beta_{i}$. Here, to simplify notation we use $c_{i}$ instead of $c\left(\alpha_{i}\right)$ and note that the $c$ dependence of $\alpha_{i}$ is inherited from $\beta_{i}$ that appears in condition (e) above. It is a simple matter to see that there are some $i \in\{1,2, \ldots, N\}$ such that $c_{i}>0$. Define $\Lambda=\sum_{i=1}^{N} c_{i} \delta_{\alpha_{i}}$, where

$$
\delta_{\alpha_{i}}(\alpha)=\left\{\begin{array}{lll}
1, & \text { if } \quad \alpha_{i}=\alpha \\
0, & \text { if } \quad \alpha_{i} \neq \alpha
\end{array}\right.
$$

Let $\operatorname{supp} \Lambda$ denote the support of $\Lambda(\alpha \in \operatorname{supp} \Lambda$, then $\Lambda(\{\alpha\})>0)$ and note that $\int_{\mathcal{A}} \Lambda(\mathrm{d} \alpha)=1$. For all $i$ such that $c_{i}>0$, define

$$
\lambda\left(\alpha_{i}\right)=\frac{\lambda \beta_{i}}{c_{i}} ; \quad w\left(t, \alpha_{i}\right)=\frac{p\left(t, \alpha_{i}\right)}{c_{i}} ; \quad s\left(t, \alpha_{i}\right)=\frac{q\left(t, \alpha_{i}\right)}{c_{i}} ; \quad y\left(t, \alpha_{i}\right)=\frac{r\left(t, \alpha_{i}\right)}{c_{i}} ; \quad \xi^{\prime}\left(t, \alpha_{i}\right)=\frac{\xi\left(t, \alpha_{i}\right)}{c_{i}} .
$$

Combining $(f)$ and (4.1), it follows that there exists an integrable function $K_{m}$ such that

$$
\left|s\left(t, \alpha_{i}\right)\right|+\left|y\left(t, \alpha_{i}\right)\right| \leq K_{m}(t)
$$


From the above, we conclude that, for any $i=1, \ldots, N$ corresponding to $c_{i}>0$, we have

i') $\left\|w\left(\cdot, \alpha_{i}\right)\right\|+\lambda\left(\alpha_{i}\right)=1$ and $0 \leq \lambda\left(\alpha_{i}\right) \leq 1$;

ii') $\left(\dot{w}\left(t, \alpha_{i}\right), 0, \xi(t, \alpha)\right) \in \operatorname{co} \partial_{x, u, v} H\left(t, \bar{x}\left(t, \alpha_{i}\right), w\left(t, \alpha_{i}\right), s\left(t, \alpha_{i}\right), y\left(t, \alpha_{i}\right), u(t), v(t), \alpha_{i}\right)$;

iii') $\xi(\cdot)=\sum_{i=1} \xi^{\prime}\left(\cdot, \alpha_{i}\right) c_{i}$, where

$$
\xi(\cdot)=\int_{\mathcal{A}} \xi^{\prime}(\cdot, \alpha) \Lambda(\mathrm{d} \alpha) \quad \text { and } \quad \xi(t) \in \operatorname{co} N_{V(t)}(\bar{v}(t))
$$

iv') $y(t, \alpha) \leq 0$ and

$$
\int_{\mathcal{A}} y(t, \alpha) \cdot l(t, \bar{x}(t, \alpha), \bar{u}(t), \bar{v}(t), \alpha) \Lambda(\mathrm{d} \alpha)=0
$$

$\left.\mathbf{v}^{\prime}\right)-w\left(T, \alpha_{i}\right) \in N_{C\left(\alpha_{i}\right)}\left(\bar{x}\left(T, \alpha_{i}\right)\right)+\lambda\left(\alpha_{i}\right) \partial g\left(\bar{x}\left(T, \alpha_{i}\right), \alpha_{i}\right)$.

Now rename $w, s, y$ and $\xi^{\prime}$ as $p, q, r$ and $\xi$, respectively. Recalling from $\left.i^{\prime}\right)$ that $\lambda\left(\alpha_{i}\right) \in[0,1]$, it is then an easy task to see that the conclusions of the proposition hold. The proof is complete.

Remark 4.3. Notice that when $\Lambda(\{\alpha\})>0$, then the parameter $\alpha$ is active in the final constraints or in the objective function, in the following sense

$$
g(\bar{x}(T, \alpha), \alpha)=\max _{\alpha^{\prime} \in \mathcal{A}} g\left(\bar{x}\left(T, \alpha^{\prime}\right), \alpha^{\prime}\right) \quad \text { ou } \quad \bar{x}(T, \alpha) \in \operatorname{bdy} C(\alpha) .
$$

Here bdy $C(\alpha)$ denotes the boundary of the set $C(\alpha)$.

Observe also that, since $\mathcal{A}$ is a discrete set, the applications $p(t, \cdot): \mathcal{A} \rightarrow \mathbb{R}^{n}, q(t, \cdot): \mathcal{A} \rightarrow \mathbb{R}^{m_{b}}, r(t, \cdot): \mathcal{A} \rightarrow$ $\mathbb{R}^{m_{b}}$ and $\xi(t, \cdot): \mathcal{A} \rightarrow \mathbb{R}^{m_{b}}$ are continuous (in the discrete topology) for all $t \in[S, T]$.

\subsection{Case where the Set $\mathcal{A}$ is infinite}

To extend the previous results to the case where $\mathcal{A}$ is an arbitrary compact metric space, we use a sequence of approximating problems associated with sets of finite parameters, for which the necessary conditions were previously obtained. Then with an adequate convergence analysis the results are provided for the general problem.

Now, and before proceeding, we introduce some useful definitions.

Given $\delta \geq 0$ and $\alpha \in \mathcal{A}$, define the following sets

$$
\begin{aligned}
& G_{\delta}(x, \alpha)= \begin{cases}\partial_{x} g(x, \alpha), & \text { if } g(x, \alpha) \geq \max _{\alpha^{\prime} \in \mathcal{A}} g\left(x, \alpha^{\prime}\right)-\delta, \\
\emptyset, & \text { otherwise, }\end{cases} \\
& N(x, \alpha)=\left\{\xi \in N_{C(\alpha)}(x)|| \xi \mid=1\right\} .
\end{aligned}
$$

and

$$
\begin{array}{r}
\mathcal{O}_{\delta}(\alpha):=\left\{(p(\cdot, \alpha), q(\cdot, \alpha), r(\cdot, \alpha), \xi(\cdot, \alpha)) \in W^{1,1} \times L^{1} \times L^{1} \times L^{1} \mid\right. \text { the conditions } \\
(I),(I I),(I I I) \text { and }(I V) \text { are satisfied }\}
\end{array}
$$

where 
(I) $(-\dot{p}(t, \alpha), 0, \xi(t, \alpha)) \in \bigcup_{(x, u, v) \in \Omega_{\delta}(t, \alpha)} \operatorname{co} \partial_{x, u, v} H(t, x, p, q, r, u, v, \alpha)$, a.e. $t \in[S, T]$,

(II) $-p(T, \alpha) \in \bigcup_{x \in \bar{x}(t, \alpha)+\delta B} \bigcup_{m \in[0,1]}\left((1-m) N(x, \alpha)+m \partial G_{\delta}(x, \alpha)\right)$,

(III) $r(\cdot, \alpha) \leq 0$,

(IV) there exists an integrable function $K_{m}$ such that

$$
|q(t, \alpha)|+|r(t, \alpha)| \leq K_{m}(t) .
$$

Note that $\mathcal{O}_{\delta=0}(\cdot)$ is immersed in the multifunction family $\left\{\mathcal{O}_{\delta}(\cdot) \mid \delta \geq 0\right\}$ and $\Omega_{\delta}(t, \alpha)$ is defined in (3.1).

Define also

$$
\mathcal{M}(\alpha):=\bigcap_{\delta>0} \overline{\operatorname{co}}\left(\bigcup_{\alpha^{\prime} \in B_{\mathcal{A}}(\alpha, \delta)} \mathcal{O}_{\delta}\left(\alpha^{\prime}\right)\right)
$$

where $\overline{\text { co }}$ denotes the closure of the convex envelope with respect to the product topology in $W^{1,1} \times L^{1} \times L^{1} \times L^{1}$.

Observe that $\mathcal{M}(\alpha)$ is a subset of $W^{1,1}\left([S, T] ; \mathbb{R}^{n}\right) \times L^{1}\left([S, T] ; \mathbb{R}^{m_{b}}\right) \times L^{1}\left([S, T] ; \mathbb{R}^{m_{l}}\right) \times L^{1}\left([S, T] ; \mathbb{R}^{k_{v}}\right)$. The enlargement is carried out in such a way that the new multifunction $\alpha \rightsquigarrow \mathcal{M}(\alpha)$ has closed graph and convex values.

With these new tools we now state the main result, the weak maximum principle for the minimax optimal control problem with equality and inequality constraints.

Theorem 4.4. Let $(\bar{u}, \bar{v},\{\bar{x}(\cdot ; \alpha) \mid \alpha \in \mathcal{A}\})$ be a weak minimum of the problem (PR). Assume that, for some $\epsilon>0$, the hypotheses S1) - S9) and A1) (or A2) or (MFC)) are satisfied. Then there exists a family of multipliers consisting of a Radon probability measure $\Lambda \in C^{*}(\mathcal{A})$ and family of functions

$$
\begin{gathered}
\left\{p(\cdot, \alpha) \in W^{1,1}\left([S, T] ; \mathbb{R}^{n}\right)\right\},\left\{q(\cdot, \alpha) \in L^{1}\left([S, T] ; \mathbb{R}^{m_{b}}\right)\right\}, \\
\left\{r(\cdot, \alpha) \in L^{1}\left([S, T] ; \mathbb{R}^{m_{l}}\right)\right\},\left\{\xi(\cdot, \alpha) \in L^{1}\left([S, T] ; \mathbb{R}^{k_{v}}\right)\right\}
\end{gathered}
$$

such that

$$
\begin{gathered}
(p(\cdot, \alpha), q(\cdot, \alpha), r(\cdot, \alpha), \xi(\cdot, \alpha)) \in \mathcal{M}(\alpha) \quad \text { para } \quad \Lambda-\text { a.e. } \quad \alpha \in \mathcal{A}, \\
\int_{\mathcal{A}} \xi(t, \alpha) \mathrm{d} \Lambda(\alpha) \in \operatorname{co} N_{V(t)}(\bar{v}(t))
\end{gathered}
$$

and

$$
\int_{\mathcal{A}} r(t, \alpha) \cdot l(t, \bar{x}(t, \alpha), \bar{u}(t), \bar{v}(t), \alpha) \mathrm{d} \Lambda(\alpha)=0 \quad \text { a.e. } \quad t \in[S, T] .
$$

Furthermore, for some integrable function $k_{m}$,

$$
\int_{\mathcal{A}}(|q(t, \alpha)|+|r(t, \alpha)|) \mathrm{d} \Lambda(\alpha) \leq k_{m}(t) \int_{\mathcal{A}}|p(t, \alpha)| \mathrm{d} \Lambda(\alpha) \quad \text { a.e. } \quad t \in[S, T] .
$$

Notice that the non triviality of the multipliers is guaranteed since the measure $\Lambda$ is a probability measure, meaning $\int_{\mathcal{A}} \Lambda(\mathrm{d} \alpha)=1$. We also point out that the conclusions of Propostion 4.2 can be expressed in the form of those in Theorem 4.4 with the multipliers $(p(\cdot, \alpha), q(\cdot, \alpha), r(\cdot, \alpha), \xi(\cdot, \alpha)) \in \mathcal{O}_{0}(\alpha)$ instead of $\mathcal{M}(\alpha)$. 
The proof of Theorem 4.4 is postponed to Section 5. We now present two examples. The first one shows that Proposition 4.2 does not hold if the set of parameters $\mathcal{A}$ is infinite. Then, in the second example, we present a problem satisfying the Mangasarian-Fromovitz constraint but not the full rankness conditions A1) and A2).

Example 4.5. Consider the following minimax optimal control problem with equality and inequality constraints

$$
\left(P R_{1}\right)\left\{\begin{array}{l}
\text { Minimize } \max _{\alpha \in[-1,1]}-|x(1)-\alpha| \text { such that } \\
\dot{x}(t)=u_{1}(t) \\
u_{1}(t)-x^{2}(t)+u_{2}(t)=0 \\
u_{2}(t)-u_{1}^{3}(t)-1 \leq 0 \\
x(0)=0
\end{array}\right.
$$

The parameters set is the interval $\mathcal{A}=[-1,1]$. The cost function depends on $\alpha$, but neither the dynamics, nor the equality and inequality constraints, depend on $\alpha$. The controls are free as well as the endpoint states. In this case, the control variable is $\left(u_{1}, u_{2}\right)$.

A minimum for the problem $\left(P R_{1}\right)$ is $\left(\bar{x}, \bar{u}_{1}, \bar{u}_{2}\right)=(0,0,0)$. We also have

$$
H\left(t, x, p, q, r, u_{1}, u_{2}\right)=p u_{1}+q\left(u_{1}-x^{2}+u_{2}\right)+r\left(u_{2}-u_{1}^{3}\right),
$$

$q_{a}(t)=0, F(t)=(1,1)$ and $\operatorname{det} F(t) F(t)^{\top}=2$. Ignoring the nature of the set $\mathcal{A}$, it is an easy matter to see that the data of this problem satisfies the hypotheses under which Proposition 4.2 holds. Applying it to $\left(P R_{1}\right)$ we get the existence of a probability measure $\Lambda$, with support in

$$
\left\{\alpha \in \mathcal{A}|-| \bar{x}(1)-\alpha \mid=\max _{\alpha^{\prime} \in[-1,1]}\left(-\left|\bar{x}(1)-\alpha^{\prime}\right|\right)\right\}=\{0\},
$$

and families of functions $\{p(\cdot, \alpha) \mid \alpha \in \mathcal{A}\},\{q(\cdot, \alpha) \mid \alpha \in \mathcal{A}\},\{r(\cdot, \alpha) \mid \alpha \in \mathcal{A}\}$ and $\xi \in L^{1}$ obeying all the conditins of Proposition 4.2. From (4.3) we have $\Lambda=\delta_{\{0\}}$, which implies that $\operatorname{supp}\{\Lambda\}=\{0\}$ and the only relevant value of $\alpha$ is $\alpha=0$. Making some calculations we may arrive at

$$
\begin{gathered}
\left\{(p, q, r, \xi) \in W^{1,1} \times L^{1} \times L^{1} \times L^{1} \mid-\dot{p}=0,-p(1) \in\{1\} \cup\{-1\}, q=-p, r=p\right\} \\
\{(p, q, r, \xi) \equiv(1,-1,1,0)\} \cup\{(p, q, r, \xi) \equiv(-1,1,-1,0)\} .
\end{gathered}
$$

Since $r(t, \alpha)$ has to be negative, we have $(p, q, r, \xi)=(-1,1,-1,0)$. Therefore,

$$
\int_{\mathcal{A}} r(t ; \alpha) l\left(t, \bar{x}(t), \bar{u}_{1}, \bar{u}_{2}\right) \Lambda(\mathrm{d} \alpha)=-1 \neq 0,
$$

showing that the assertions of Proposition 4.2 fail. The pathology is the fact that $\mathcal{A}$ is infinite.

Notice, however, that the minimum $\left(\bar{x}, \bar{u}_{1}, \bar{u}_{2}\right)=(0,0,0)$ of problem (PR1) satisfies all the conditions of Theorem 4.4.

Example 4.6. Consider the following minimax optimal control problem with equality and inequality constraints

$$
\left(P R_{2}\right)\left\{\begin{array}{l}
\text { Minimize } \max _{\alpha \in[-0.5,1]}-|x(1)-\alpha| \text { such that } \\
\dot{x}(t)=u_{1}(t) \\
u_{1}^{2}(t)+u_{2}(t)=0 \\
u_{1}(t)+\alpha x(t)+\alpha u_{2}^{2}(t) \leq 0 \\
(1+\alpha) u_{1}(t) \leq 0, \quad x(0)=0 .
\end{array}\right.
$$


The parameters set is the interval $\mathcal{A}=[-0.5,1]$. Note that this is a slight modification of the previous example on the constraints. The controls are free as well as the endpoint states. In this case, the control variable is $\left(u_{1}, u_{2}\right)$. A simple observation of the problem shows that the minimum is $\left(\bar{x}, \bar{u}_{1}, \bar{u}_{2}\right)=(0,0,0)$. We have

$$
\begin{aligned}
b\left(t, x, u_{1}, u_{2}, \alpha\right) & =u_{1}^{2}(t)+u_{2}(t), & \nabla_{u} b\left(t, x, u_{1}, u_{2}, \alpha\right) & =\left(2 u_{1}, 1\right), \\
l_{1}\left(t, x, u_{1}, u_{2}, \alpha\right) & =u_{1}(t)+\alpha x(t)+\alpha u_{2}^{2}(t), & & \nabla_{u} l_{1}\left(t, x, u_{1}, u_{2}, \alpha\right)=\left(1,2 \alpha u_{2}(t)\right), \\
l_{2}\left(t, x, u_{1}, u_{2}, \alpha\right) & =(1+\alpha) u_{1}(t), & & \nabla_{u} l_{2}\left(t, x, u_{1}, u_{2}, \alpha\right)=(1+\alpha, 0)
\end{aligned}
$$

and, consequently, for the optimal solution

$$
F^{T}(t)=\left(\begin{array}{ccc}
0 & 1 & 1+\alpha \\
1 & 0 & 0
\end{array}\right)
$$

and $\operatorname{det} F(t) F(t)^{\top}=0$. That means that Problem $\left(P R_{2}\right)$ does not satisfy the full rank condition. However, by taking $h(t)=(1,0)^{T}, a(t)=(1,1+\alpha)^{T}, K_{1}=0.4$ and $K_{2}=0.9>0$ we see that the Mangassarian-Fromovitz condition (MFC) is valid. Then by Theorem 4.4 we have that there are $(p(\cdot, \alpha), q(\cdot, \alpha), r(\cdot, \alpha), \xi(\cdot, \alpha)) \in \mathcal{M}(\alpha)$ for $\Lambda$ - a.e. $\alpha \in \mathcal{A}$, such that

$$
\int_{\mathcal{A}} r(t, \alpha) \cdot l(t, \bar{x}(t, \alpha), \bar{u}(t), \bar{v}(t), \alpha) \mathrm{d} \Lambda(\alpha)=0
$$

and

$$
\int_{\mathcal{A}} \xi(t, \alpha) \mathrm{d} \Lambda(\alpha) \in \operatorname{co} N_{V(t)}(\bar{v}(t))
$$

where $H\left(t, x, p, q, r, u_{1}, u_{2}, \alpha\right)=p u_{1}+q\left(u_{1}^{2}+u_{2}\right)+r_{1}\left(u_{1}+\alpha x+\alpha u_{2}^{2}\right)+r_{2}(1+\alpha) u_{1}$ and $\partial_{x, u, v} H\left(t, x, p, q, r, u_{1}, u_{2}, \alpha\right)=$ $\left(r_{1} \alpha, p+2 q u_{1}+r_{1}+r_{2}(1+\alpha), q+2 \alpha r_{1} u_{2}\right)$ and $\partial_{x, u, v} H\left(t, \bar{x}, p, q, r, u_{1}, u_{2}, \alpha\right)=\left(r_{1} \alpha, p+r_{1}+r_{2}(1+\alpha), q\right)$.

Since $(p, q, r, \xi) \in M(\alpha)$ we have

(i) $(-\dot{p}, 0,0)=\left(r_{1} \alpha, p+r_{1}+r_{2}(1+\alpha), q\right)$, from where $-\dot{p}=r_{1} \alpha, \quad 0=p+r_{1}+r_{2}(1+\alpha) \quad$ and $\quad 0=q$;

(ii) $-p(1) \in\{1\} \cup\{-1\}$;

(iii) $r_{1} \leq 0$ and $r_{2} \leq 0$.

Due to $-\dot{p}(t, \alpha)=r_{1} \alpha$, we obtain $p(t, \alpha)=r_{1} \alpha+p(1, \alpha)$.

- If $p(1, \alpha)=1$, then $p(t, \alpha)=r_{1} \alpha+1$. Replacing in $(i)$, we have $0=r_{1} \alpha+1+r_{1}+r_{2}(1+\alpha)$, from where $-\frac{1}{\alpha+1}-r_{1}=r_{2} \leq 0$. Then we have $r_{1} \geq-\frac{1}{1+\alpha}$.

- If $p(1, \alpha)=-1$, then $p(t, \alpha)=r_{1} \alpha-1$. Replacing in $(i)$, we have $0=r_{1} \alpha-1+r_{1}+r_{2}(1+\alpha)$, from where $\frac{1}{\alpha+1}-r_{1}=r_{2} \leq 0$. Then we have $r_{1} \geq \frac{1}{1+\alpha}$ and, since $r_{1} \leq 0$, we have $0 \geq \frac{1}{1+\alpha}$. This is impossible since $\alpha \in[-0.5,1]$.

Thus, $p(1, \alpha)=1$. It follows that $(p(\cdot, \alpha), q(\cdot, \alpha), r(\cdot, \alpha), \xi(\cdot, \alpha)) \in\left\{\left(r_{1} \alpha+1,0, r, 0\right) / r=\left(r_{1}, r_{2}\right), r_{1}+r_{2}=\right.$ $\left.-\frac{1}{1+\alpha}, r_{1} \in[-2,0]\right\}$.

\section{Proof of the Theorem 4.4}

Without loss of generality, we prove Theorem 4.4, replacing the hypotheses S1), S2), S3), S5) and S6) by stronger ones by setting $\epsilon=+\infty$. This means that all mentioned hypotheses remain valid for all $x, x^{\prime} \in \mathbb{R}^{n}$, $u, u^{\prime} \in \mathbb{R}^{k_{u}}$ and $v, v^{\prime} \in V(t)$, not merely in $\Omega_{\epsilon}(t, \alpha)$. This can be achivied by replacing $f, b, l$ and $g$ by

$$
(t, x, u, v, \alpha) \mapsto f\left(t, \bar{x}(t, \alpha)+\operatorname{tr}_{\delta}(x-\bar{x}(t, \alpha)), \bar{u}(t)+\operatorname{tr}_{\delta}(u-\bar{u}(t)), \bar{v}(t)+\operatorname{tr}_{\delta}(v-\bar{v}(t)), \alpha\right),
$$




$$
\begin{aligned}
& (t, x, u, v, \alpha) \mapsto b\left(t, \bar{x}(t, \alpha)+\operatorname{tr}_{\delta}(x-\bar{x}(t, \alpha)), \bar{u}(t)+\operatorname{tr}_{\delta}(u-\bar{u}(t)), \bar{v}(t)+\operatorname{tr}_{\delta}(v-\bar{v}(t)), \alpha\right), \\
& \left.(t, x, u, v, \alpha) \mapsto l\left(t, \bar{x}(t, \alpha)+\operatorname{tr}_{\delta}(x-\bar{x}(t, \alpha)), \bar{u}(t)+\operatorname{tr}_{\delta}(u-\bar{u}(t)), \bar{v}(t)+\operatorname{tr}_{\delta}(v-\bar{v}(t)), \alpha\right)\right)
\end{aligned}
$$

and

$$
(x, \alpha) \mapsto g\left(\bar{x}(t, \alpha)+\operatorname{tr}_{\delta}(x-\bar{x}(t, \alpha)), \alpha\right) .
$$

The property that $\bar{x}$ is a weak minimizer of the problem $(\mathrm{PR})$ is preserved under these strengthened hypotheses, as well as the fact that for a given $w=(u, v) \in \mathcal{W}$ and $\alpha \in \mathcal{A}$, there corresponds a unique state trajectory satisfying the dynamics of problem (PR)), which we denote by $x(\cdot, \alpha, u, v)$.

Denote by $\bar{w}:=(\bar{u}, \bar{v}) \in \mathcal{W}(t)$. Let's take a decreasing sequence $\delta_{i} \downarrow 0$, as $i \rightarrow \infty$. Since $(\bar{u}, \bar{v},\{\bar{x}(\cdot ; \alpha) \mid \alpha \in \mathcal{A}\})$ is a weak minimum of the problem $(\mathrm{PR})$, then

$$
\max _{\alpha \in \mathcal{A}} g(x(T, \alpha), \alpha) \geq \max _{\alpha \in \mathcal{A}} g(\bar{x}(T, \alpha), \alpha) .
$$

Suppose, without loss of generality, that $\max _{\alpha \in \mathcal{A}} g(\bar{x}(T, \alpha), \alpha)=0$ and let $\left\{\alpha_{i}\right\}_{i=1}^{\infty}$ be a dense enumerable set in $\mathcal{A}$. Since $\mathcal{A}$ a compact metric space, for any $i \in\{1,2, \ldots\}$, there exists an integer $N=N(i)$ such that

$$
\max _{\alpha \in \mathcal{A}_{N}} g(x(T, \alpha), \alpha) \geq \max _{\alpha \in \mathcal{A}} g(x(T, \alpha), \alpha)-\delta_{i}^{2},
$$

where $\mathcal{A}_{N}:=\left\{\alpha_{1}, \ldots, \alpha_{N}\right\}$ is chosen such that $\mathcal{A}_{N(i)} \subset \mathcal{A}_{N(i+1)}$.

Fix any $i \in\{1,2, \ldots\}$ and define

$$
J_{i}(u, v):=\max _{\alpha \in \mathcal{A}_{N}} \max \left\{g(x(T, \alpha, u, v), \alpha)+\delta_{i}^{2}, d_{C_{\alpha}}(x(T, \alpha, u, v))\right\} .
$$

Notice that $J_{i}$ is continuous with respect to $\Delta$-metric topology on $\mathcal{W}, J_{i}(u, v) \geq 0$ for all $(u, v) \in \mathcal{W}$ and $J_{i}(\bar{u}, \bar{v})=\delta_{i}^{2}$. So $(\bar{u}, \bar{v})$ is a $\delta_{i}^{2}$ minimizing for the functional $J_{i}$ over $\mathcal{W}$. It follows from Ekeland's Variational Principle ([18], Thm. 3.3.1) that there exists a control function $\left(u_{i}, v_{i}\right)$ such that $\Delta\left(\left(u_{i}, v_{i}\right),(\bar{u}, \bar{v})\right) \leq \delta_{i}$ and

$$
J_{i}\left(u_{i}, v_{i}\right)+\delta_{i} \Delta\left(\left(u_{i}, v_{i}\right),\left(u_{i}, v_{i}\right)\right)=\min _{(u, v) \in \mathcal{W}}\left\{J_{i}(u, v)+\delta_{i} \Delta\left(\left(u_{i}, v_{i}\right),(u, v)\right)\right\} .
$$

We have $J_{i}\left(u_{i}, v_{i}\right)>0$, for all $i$ sufficiently large. In fact, if $J_{i}\left(u_{i}, v_{i}\right)=0$ for some $i$, since the $J_{i}$ are nonnegative, we must have

$$
x\left(T, \alpha, u_{i}, v_{i}\right) \in C(\alpha) \quad \text { and } \quad g\left(x\left(T, \alpha, u_{i}, v_{i}\right), \alpha\right)+\delta_{i}^{2}=0
$$

for all $\alpha \in \mathcal{A}_{N}$. Lemma 3.1 and continuity properties of $g$ imply that

$$
g\left(x\left(T, \alpha, u_{i}, v_{i}\right), \alpha\right)<g\left(x\left(T, \alpha, u_{i}, v_{i}\right), \alpha\right)+\delta_{i}^{2}=0=\max _{\alpha \in \mathcal{A}} g(\bar{x}(T, \alpha), \alpha),
$$

violating the optimality of $(\bar{u}, \bar{v},\{\bar{x}(\cdot ; \alpha) \mid \alpha \in \mathcal{A}\})$.

Summarizing, we have: for $\delta_{i} \downarrow 0$, there are a sequence $\left\{\left(u_{i}, v_{i}\right)\right\} \in \mathcal{W}$ and an increasing sequence of finite subsets $\mathcal{A}_{N}$ of $\mathcal{A}$ such that

(i) $J_{i}\left(u_{i}, v_{i}\right)+\delta_{i} \Delta\left(\left(u_{i}, v_{i}\right),\left(u_{i}, v_{i}\right)\right)=\min _{(u, v) \in \mathcal{W}}\left\{J_{i}(u, v)+\delta_{i} \Delta\left(\left(u_{i}, v_{i}\right),(u, v)\right)\right\}$; 
(ii) $J_{i}\left(u_{i}, v_{i}\right)>0$, for all $i$;

(iii) $\Delta\left(\left(u_{i}, v_{i}\right),(\bar{u}, \bar{v})\right) \rightarrow 0$ as $i \rightarrow \infty$.

Write $\left\{x_{i}(\cdot, \alpha) \mid \alpha \in \mathcal{A}_{N(i)}\right\}$ for the state trajectories corresponding to $\left(u_{i}, v_{i}\right)$. For each $i,\left(u_{i}, v_{i},\left\{x_{i}\left(\cdot, \alpha_{k}^{i}\right) \mid k=\right.\right.$ $1,2, \ldots, N(i)\})$ is a minimum for the optimal control problem $\left(P R_{i}\right)$

$$
\left(P R_{i}\right) \quad\left\{\begin{array}{l}
\text { Minimize } \max _{1 \leq k \leq N} \max \left\{g\left(x\left(T, \alpha_{k}^{i}\right), \alpha_{k}^{i}\right)+\delta_{i}^{2}, d_{C_{\alpha_{k}^{i}}}\left(x\left(T, \alpha_{k}^{i}\right), \alpha_{k}^{i}\right)\right\}+ \\
\quad \delta_{i} \int_{S}^{T}\left(\left|u(t)-u_{i}(t)\right|+\left|v(t)-v_{i}(t)\right|\right) \mathrm{d} t \\
\text { over processes }\left\{\left(u, v, x\left(\cdot, \alpha_{k}^{i}\right)\right): k=1, \cdots, N\right\} \text { such that } \\
(u, v) \in \mathcal{W}, \\
\dot{x}\left(t, \alpha_{k}^{i}\right)=f\left(t, x\left(t, \alpha_{k}^{i}\right), u(t), v(t), \alpha_{k}^{i}\right) \\
0=b\left(t, x\left(t, \alpha_{k}^{i}\right), u(t), v(t), \alpha_{k}^{i}\right) \\
0 \geq l\left(t, x\left(t, \alpha_{k}^{i}\right), u(t), v(t), \alpha_{k}^{i}\right) \\
x\left(S ; \alpha_{k}^{i}\right)=x_{0} \\
k=1,2, \ldots, N
\end{array}\right.
$$

It follows from (iii) and Lemma 3.1 that, for $\alpha \in \mathcal{A}$,

$$
\left\|x\left(\cdot, \alpha, u_{i}, v_{i}\right)-\bar{x}(\cdot, \alpha)\right\| \rightarrow 0, \text { as } i \rightarrow \infty
$$

The sequence of problems $\left(P R_{i}\right)$ can now be rewritten as

$$
\left(\widetilde{P R}_{i}\right) \quad\left\{\begin{array}{l}
\text { Minimize } \tilde{g}(x(T))+\delta_{i} \int_{S}^{T}\left(\left|u(t)-u_{i}(t)\right|+\left|v(t)-v_{i}(t)\right|\right) \mathrm{d} t \\
\text { subject to } \\
\dot{x}(t)=\tilde{f}(t, x(t), u(t), v(t)), \quad \text { a.e. } t \in[S, T] \\
0=\tilde{b}(t, x(t), u(t), v(t)), \quad \text { a.e. } t \in[S, T] \\
0 \geq \tilde{l}(t, x(t), u(t), v(t)), \quad \text { a.e. } t \in[S, T] \\
v(t) \in V(t), \\
x(S)=\tilde{x}_{0},
\end{array}\right.
$$

where

$$
\begin{aligned}
x & =\operatorname{col}\left\{x_{1}, x_{2}, \ldots, x_{N(i)}\right\}, x_{j} \in \mathbb{R}^{n}, j=1, \cdots N(i), \\
\tilde{f}(t, x, u, v) & =\operatorname{col}\left\{f\left(t, x_{j}, u, v, \alpha_{j}\right)\right\}_{j=1}^{N(i)}, \\
\tilde{b}(t, x, u, v) & =\operatorname{col}\left\{b\left(t, x_{j}, u, v, \alpha_{j}\right)\right\}_{j=1}^{N(i)}, \\
\tilde{l}(t, x, u, v) & =\operatorname{col}\left\{l\left(t, x_{j}, u, v, \alpha_{j}\right)\right\}_{j=1}^{N(i)}, \\
\tilde{x}_{0} & =\operatorname{col}\left\{x_{0}, x_{0}, \ldots, x_{0}\right\}, \\
\tilde{g}(x(\cdot)) & =\max _{1 \leq k \leq N(i)} \max \left\{g\left(x\left(T, \alpha_{k}^{i}\right), \alpha_{k}^{i}\right)+\delta_{i}^{2}, d_{C_{\alpha_{k}^{i}}}\left(x\left(T, \alpha_{k}^{i}\right), \alpha_{k}^{i}\right)\right\} .
\end{aligned}
$$

We observe that problem $\left(\widetilde{P R}_{i}\right)$ does not have endpoint constraints. So applying Proposition 4.1 with $\lambda=1$ to each problem $\left(\widetilde{P R}_{i}\right)$, together with Lemma 3.3, the Sum Rule ([18], Thm. 5.4.1) and the Maximum Rule ([18], Thm. 5.5.2) for subdifferentials, asserts the existence of multipliers $\lambda_{k}^{i}, p_{i}^{\prime}(\cdot, \alpha), q_{i}^{\prime}(\cdot, \alpha), r_{i}^{\prime}(\cdot, \alpha)$ and $\xi_{i}^{\prime}(\cdot, \alpha)$ such that 
(A) for $k=\{1, \ldots, N(i)\}$,

$$
\begin{array}{r}
\left(-\dot{p}_{i}^{\prime}\left(t, \alpha_{k}^{i}\right), 0, \xi_{i}^{\prime}\left(t, \alpha_{k}^{i}\right)\right) \in \operatorname{co} \partial_{x, u, v} H\left(t, x_{i}\left(t, \alpha_{k}^{i}\right), p_{i}^{\prime}\left(t, \alpha_{k}^{i}\right), q_{i}^{\prime}\left(t, \alpha_{k}^{i}\right), r_{i}^{\prime}\left(t, \alpha_{k}^{i}\right), u_{i}(t), v_{i}(t), \alpha_{k}^{i}\right) \\
-\left(\{0\} \times \delta_{i} \mathbb{B} \times \delta_{i} \mathbb{B},\right) \quad \text { a.e. } \quad t \in[S, T] .
\end{array}
$$

(B) $\xi_{i}^{\prime}(t)=\sum_{k=1}^{N(i)} \xi_{i}^{\prime}\left(t, \alpha_{k}^{i}\right) \in \operatorname{co} N_{V(t)}\left(v_{i}(t)\right)$

(C) for $k=\{1, \ldots, N(i)\},-p_{i}^{\prime}\left(T, \alpha_{k}^{i}\right) \in \lambda_{k}^{i} \partial g\left(x_{i}\left(T, \alpha_{k}^{i}\right), \alpha_{k}^{i}\right)+b_{k}^{i}$, where $b_{k}^{i} \in N_{C_{\alpha}}\left(x_{i}\left(T, \alpha_{k}^{i}\right)\right)$ and $\sum_{k=1}^{N(i)} \lambda_{k}^{i}+\left|b_{k}^{i}\right|=$ 1.

(D) $\sum_{k=1}^{N(i)} r_{i}^{\prime}\left(t, \alpha_{k}^{i}\right) l\left(t, x_{i}\left(t, \alpha_{k}^{i}\right), u_{i}(t), v_{i}(t), \alpha_{k}^{i}\right)=0$ e $r_{i}^{\prime}\left(t, \alpha_{k}^{i}\right) \leq 0$ for all $1 \leq k \leq N(i)$, a.e. $t \in[S, T]$.

(E) For each $i \in \mathbb{N}$, there exists an integrable function $K_{m}^{i}$ such that

$$
\sum_{k=1}^{N(i)}\left(\left|q_{i}^{\prime}\left(t, \alpha_{k}^{i}\right)\right|+\left|r_{i}^{\prime}\left(t, \alpha_{k}^{i}\right)\right|\right) \leq K_{m}^{i}(t) \sum_{k=1}^{N(i)}\left|p_{i}^{\prime}\left(t, \alpha_{k}^{i}\right)\right| \quad \text { a.e. } \quad t \in[S, T]
$$

Define the following probability measure on the Borel subsets of $\mathcal{A}$ by

$$
\Lambda_{i}=\sum_{k=1}^{N(i)}\left(\lambda_{k}^{i}+\left|b_{k}^{i}\right|\right) \delta_{\alpha_{k}^{i}}
$$

Next, we redefine the above family of multipliers by

$$
p_{i}\left(t, \alpha_{k}^{i}\right)=\frac{p_{i}^{\prime}\left(t, \alpha_{k}^{i}\right)}{\left|b_{k}^{i}\right|+\lambda_{k}^{i}} ; \quad q_{i}\left(t, \alpha_{k}^{i}\right)=\frac{q_{i}^{\prime}\left(t, \alpha_{k}^{i}\right)}{\left|b_{k}^{i}\right|+\lambda_{k}^{i}} ; \quad r_{i}\left(t, \alpha_{k}^{i}\right)=\frac{r^{\prime}\left(t, \alpha_{k}^{i}\right)}{\left|b_{k}^{i}\right|+\lambda_{k}^{i}} ; \quad \xi_{i}\left(t, \alpha_{k}^{i}\right)=\frac{\xi_{i}^{\prime}\left(t, \alpha_{k}^{i}\right)}{\left|b_{k}^{i}\right|+\lambda_{k}^{i}},
$$

when $\alpha_{k}^{i} \in \operatorname{supp} \Lambda_{i}$ for some $k \in\{1, \ldots, N(i)\}$. Observe that $\left(\lambda_{k}^{i}+\left|b_{k}^{i}\right|\right)>0$.

It then follows from (A)-(E) above that

(A2) for each $\alpha_{k}^{i} \in \operatorname{supp} \Lambda_{i}$,

$$
\begin{gathered}
\left(-\dot{p}_{i}\left(t, \alpha_{k}^{i}\right), 0, \xi_{i}\left(t, \alpha_{k}^{i}\right)\right) \in \operatorname{co} \partial_{x, u, v} H\left(t, x_{i}\left(t, \alpha_{k}^{i}\right), p_{i}\left(t, \alpha_{k}^{i}\right), q_{i}\left(t, \alpha_{k}^{i}\right), r_{i}\left(t, \alpha_{k}^{i}\right), u_{i}(t), v_{i}(t), \alpha_{k}^{i}\right) \\
-\left(\{0\} \times \delta_{i} \mathbb{B} \times \delta_{i} \mathbb{B},\right) \quad \text { a.e. } \quad t \in[S, T] .
\end{gathered}
$$

(B2) $\xi_{i}(t)=\int_{\mathcal{A}_{N}} \xi_{i}(t, \alpha) d \Lambda_{i}(\alpha), \quad \xi_{i}(t) \in \operatorname{co} N_{V(t)}\left(v_{i}(t)\right)$

(C2) for each $\alpha_{k}^{i} \in \operatorname{supp} \Lambda_{i}$,

$$
-p_{i}\left(T, \alpha_{k}^{i}\right) \in m_{k}^{i} \partial g\left(x_{i}\left(T, \alpha_{k}^{i}\right), \alpha_{k}^{i}\right)+\left(1-m_{k}^{i}\right)\left\{\xi \in N_{C_{\alpha}}\left(x_{i}\left(T, \alpha_{k}\right)\right):|\xi|=1\right\},
$$

where $m_{k}^{i}=\frac{\lambda_{k}^{i}}{\lambda_{k}^{i}+\left|b_{k}^{i}\right|}$.

(D2) $\int_{\mathcal{A}_{N}} r_{i}(t, \alpha) l\left(t, x_{i}(t, \alpha), u_{i}(t), v_{i}(t), \alpha\right) \mathrm{d} \Lambda_{i}(\alpha)=0$ and $r_{i}(t, \alpha) \leq 0$ a.e. $t \in[S, T]$. 
(E2) For each $i \in \mathbb{N}$, there exists an integrable function $K_{m}^{i}$ such that

$$
\int_{\mathcal{A}_{N}}\left(\left|q_{i}(t, \alpha)\right|+\left|r_{i}(t, \alpha)\right|\right) \mathrm{d} \Lambda_{i}(\alpha) \leq K_{m}^{i}(t) \int_{\mathcal{A}_{N}}\left|p_{i}(t, \alpha)\right| \mathrm{d} \Lambda_{i}(\alpha) \quad \text { a.e. } \quad t \in[S, T]
$$

and

$$
\left|q_{i}(t, \alpha)\right|+\left|r_{i}(t, \alpha)\right| \leq K_{m}^{i}(t) \int_{\mathcal{A}_{N}}\left|p_{i}(t, \alpha)\right| \mathrm{d} \Lambda_{i}(\alpha), \forall \alpha \in A_{N}
$$

Note that item $(C 2)$ can be rewritten as

$$
-p_{i}(T, \alpha) \in \bigcup_{r \in[0,1]}\left((1-r) N\left(x_{i}(T, \alpha), \alpha\right)+r \partial G_{0}\left(x_{i}(T, \alpha), \alpha\right)\right) .
$$

Since $H$ is a Lipschitz function in the variables $(x, u, v)$, we appeal to Theorem 4.6 .2 of [18] and $(A 2)$ to conclude that

$$
\left|\dot{p}_{i}\left(t, \alpha_{k}^{i}\right)\right| \leq\left|p_{i}\left(t, \alpha_{k}^{i}\right)\right| k_{f}(t)+\left(\left|q_{i}\left(t, \alpha_{k}^{i}\right)\right|+\left|r_{i}\left(t, \alpha_{k}^{i}\right)\right|\right) L_{b, l}(t), \quad \text { for all } i \in \mathbb{N}
$$

On the other hand, by the Gronwall's inequality, we have

$$
\left|p_{i}\left(t, \alpha_{k}^{i}\right)\right| \leq \exp \left(\left\|k_{f}\right\|_{1}\right)\left(\left|p_{i}\left(T, \alpha_{k}^{i}\right)\right|+\|v\|_{1}\right)
$$

where $v(t)=\left(\left|q_{i}\left(t, \alpha_{k}^{i}\right)\right|+\left|r_{i}\left(t, \alpha_{k}^{i}\right)\right|\right) L_{b, l}(t)$. From this last inequality and (5.2), we deduce that there exists $M>0$ such that

$$
\left\|p_{i}\left(\cdot, \alpha_{k}^{i}\right)\right\|_{\infty} \leq M, \quad \text { for all } i \in \mathbb{N} .
$$

It follows from the above and $(E 2)$ that there exists integrable function $\bar{K}^{i}$ such that

$$
\left|q_{i}(t, \alpha)\right|+\left|r_{i}(t, \alpha)\right| \leq \bar{K}^{i}(t) \quad \text { a.e. } t \in[S, T] \text { and } \alpha \in \mathcal{A}_{N}
$$

From (5.2), we obtain

$$
\operatorname{supp} \Lambda_{i} \subset\left\{\alpha \in \mathcal{A}_{N(i)}: G_{0}\left(x_{i}(T, \alpha), \alpha\right) \neq \emptyset \quad \text { ou } \quad N\left(x_{i}(T, \alpha), \alpha\right) \neq \emptyset\right\} .
$$

Since (5.1) and $(A 2)-(E 2)$ hold, we can appropriately choose a subsequence $\left\{\delta_{i}^{\prime}\right\} \downarrow 0$ of $\left\{\delta_{i}\right\}$ such that, for each $\alpha \in \mathcal{A}$,

(a') $\left(-\dot{p}_{i}(t, \alpha), 0, \xi_{i}(t, \alpha)\right) \in \operatorname{co} \partial_{x, u, v} H\left(t, \bar{x}(t, \alpha)+\delta_{i}^{\prime} \mathbb{B}, p_{i}(t, \alpha), q_{i}(t, \alpha), r_{i}(t, \alpha), \bar{u}(t)+\delta_{i}^{\prime} \mathbb{B}, \bar{v}(t)+\delta_{i}^{\prime} \mathbb{B}, \alpha\right)-(\{0\} \times$ $\left.\delta_{i} \mathbb{B} \times \delta_{i} \mathbb{B}\right)$, a.e. $t \in[S, T]$.

(b') $\xi_{i}(t)=\int_{\mathcal{A}} \xi_{i}(t, \alpha) \mathrm{d} \Lambda_{i}(\alpha), \xi_{i}(t) \in \operatorname{co} N_{V(t)}\left(v_{i}(t)\right)$.

(c')

$$
-p_{i}(T, \alpha) \in \bigcup_{x \in \bar{x}(T, \alpha)+\delta_{i}^{\prime} \mathbb{B} B} \bigcup_{r \in[0,1]}\left((1-r) N(x, \alpha)+r \partial G_{\delta_{i}^{\prime}}(x, \alpha)\right) .
$$


(d') $r_{i}(t, \alpha) \leq 0$ a.e. $t \in[S, T]$

$$
0 \leq \int_{\mathcal{A}} r_{i}(t, \alpha) l(t, \bar{x}(t, \alpha), \bar{u}(t), \bar{v}(t), \alpha) \mathrm{d} \Lambda_{i}(\alpha) \leq \delta_{i}^{\prime} \quad \text { a.e. } \quad t \in[S, T]
$$

(e') For some integrable function $K_{m}^{i}$, we have

$$
\int_{\mathcal{A}}\left(\left|q_{i}(t, \alpha)\right|+\left|r_{i}(t, \alpha)\right|-k_{m}(t)\left|p_{i}(t, \alpha)\right|\right) d \Lambda_{i}(\alpha) \leq \delta_{i}^{\prime} \quad \text { a.e. } \quad t \in[S, T] .
$$

Since $\Lambda_{i}$ is a sequence of Radon probability measures on Borel sets of $\mathcal{A}$, it has a weakly* convergent subsequence (we do not re-lable). So we may write,

$$
\Lambda_{i} \rightarrow \Lambda \text { weakly* as } i \rightarrow \infty
$$

to some Radon probability measure $\Lambda$ on Borel sets of $\mathcal{A}$.

Definition 5.1. Fix a natural number $M$. Let $\gamma_{j}=\left(a_{j}, b_{j}, c_{j}\right), j=1, \ldots, M$. Define the following multifunctions $\mathfrak{T}_{i}, \mathfrak{T}: \mathcal{A} \rightarrow \mathbb{R}^{3 \times M}$ such that, for each $\alpha \in A$, their images are given by the sets

$$
\begin{aligned}
& \mathfrak{T}_{i}(\alpha)=\left\{\Gamma_{M}^{i}(\alpha)=\left(\gamma_{1}, \ldots, \gamma_{M}\right) \mid \quad \exists(p(\cdot, \alpha), q(\cdot, \alpha), r(\cdot, \alpha), \xi(\cdot, \alpha)) \in \mathcal{O}_{\delta_{i}^{\prime}}(\alpha) \quad\right. \text { such that } \\
& a_{j}=w_{j}(r(\cdot, \alpha), \alpha)=\int_{S}^{T} r(t, \alpha) \cdot l(t, \bar{x}(t, \alpha), \bar{u}(t), \bar{v}(t), \alpha) \mathrm{d} t, \\
& b_{j}=z_{j}(\xi(\cdot, \alpha), \alpha)=\int_{S}^{T} \xi(t, \alpha) \mathrm{d} t \\
& \left.c_{j}=w_{j}(p(\cdot, \alpha), q(\cdot, \alpha), r(\cdot, \alpha), \alpha)=\int_{S}^{T}\left(|q(t, \alpha)|+|r(t, \alpha)|-k_{m}(t)|p(t, \alpha)|\right) \mathrm{d} t\right\}
\end{aligned}
$$

and

$$
\begin{gathered}
\mathfrak{T}(\alpha)=\left\{\Gamma_{M}(\alpha)=\left(\gamma_{1}, \ldots, \gamma_{M}\right) \mid \quad \exists(p(\cdot, \alpha), q(\cdot, \alpha), r(\cdot, \alpha), \xi(\cdot, \alpha)) \in \mathcal{M}(\alpha) \quad\right. \text { such that } \\
a_{j}=w_{j}(r(\cdot, \alpha), \alpha)=\int_{S}^{T} r(t, \alpha) \cdot l(t, \bar{x}(t, \alpha), \bar{u}(t), \bar{v}(t), \alpha) \mathrm{d} t, \\
b_{j}=z_{j}(\xi(\cdot, \alpha), \alpha)=\int_{S}^{T} \xi(t, \alpha) \mathrm{d} t, \\
\left.c_{j}=w_{j}(p(\cdot, \alpha), q(\cdot, \alpha), r(\cdot, \alpha), \alpha)=\int_{S}^{T}\left(|q(t, \alpha)|+|r(t, \alpha)|-k_{m}(t)|p(t, \alpha)|\right) \mathrm{d} t\right\} .
\end{gathered}
$$

We summarise as Lemma 5.2 the properties of the multifunctions $\mathfrak{T}_{i}$, and $\mathfrak{T}$ that will be need in the sequel.

Lemma 5.2. The multifunctions $\mathfrak{T}_{i}, \mathfrak{T}: \mathcal{A} \rightarrow \mathbb{R}^{3 \times M}$ satisfy: a) for each $\alpha \in A, \mathfrak{T}(\alpha)$ is convex, $\left.\boldsymbol{b}\right)$ the graph of $\mathfrak{T}$, denoted by $\operatorname{Gr} \mathfrak{T}$, is compact and $\boldsymbol{c}) \lim \sup \operatorname{Gr} \mathfrak{T}_{i} \subset \operatorname{Gr} \mathfrak{T}$.

Proof. The proof of assertion a) is immediate. The proofs of b) and c) follow similar lines, so we only prove c). Take $\left(\alpha_{0}, x_{0}\right) \in \limsup \operatorname{Gr} \mathfrak{T}_{i}$. It follows from the definition of limsup that there exists a subsequence $\left\{\operatorname{Gr} \mathfrak{T}_{i_{k}}\right\} \subset\left\{\operatorname{Gr} \mathfrak{T}_{i}\right\}$ of $\left\{\operatorname{Gr} \mathfrak{T}_{i}\right\}$ and $\left(x_{k}, \alpha_{k}\right) \in \operatorname{Gr} \mathfrak{T}_{i_{k}}$ such that $\left(\alpha_{k}, x_{k}\right) \rightarrow\left(\alpha_{0}, x_{0}\right)$. Since $x_{k} \in \mathfrak{T}_{i_{k}}\left(\alpha_{k}\right)$, there 
exists $\left(p\left(\cdot, \alpha_{k}\right), q\left(\cdot, \alpha_{k}\right), r\left(\cdot, \alpha_{k}\right), \xi\left(\cdot, \alpha_{k}\right)\right) \in \mathcal{O}_{\delta_{k}^{\prime}}\left(\alpha_{k}\right)$ satisfying

$$
\begin{aligned}
a_{j}^{k} & =\int_{S}^{T} r_{k}(t) \cdot l\left(t, \bar{x}\left(t, \alpha_{k}\right), \bar{u}(t), \bar{v}(t), \alpha_{k}\right) \mathrm{d} t, \\
b_{j}^{k} & =\int_{S}^{T} \xi_{k}(t) \mathrm{d} t, \\
c_{j}^{k} & =\int_{S}^{T}\left(\left|q_{k}(t)\right|+\left|r_{k}(t)\right|-k_{m}(t)\left|p_{k}(t)\right|\right) \mathrm{d} t,
\end{aligned}
$$

where we changed notation by making $p_{k}(\cdot):=p\left(\cdot ; \alpha_{k}\right), q_{k}(\cdot):=q\left(\cdot ; \alpha_{k}\right), r_{k}(\cdot):=r\left(\cdot ; \alpha_{k}\right)$ and $\xi_{k}(\cdot):=\xi\left(\cdot ; \alpha_{k}\right)$. By the definition of $\mathcal{O}_{\delta_{k}^{\prime}}\left(\alpha_{k}\right)$, we can write

(a') $\left(-\dot{p}_{k}(t), 0, \xi_{k}(t)\right) \in \operatorname{co} \partial_{x, u, v} H\left(t, \bar{x}\left(t, \alpha_{k}\right)+\delta_{k}^{\prime} \mathbb{B}, p_{k}(t), q_{k}(t), r_{k}(t), \bar{u}(t)+\delta_{k}^{\prime} \mathbb{B}, \bar{v}(t)+\delta_{k}^{\prime} \mathbb{B}, \alpha_{k}\right), \quad$ a.e. $t \in$ $[S, T]$

(b') $-p_{k}(T) \in \bigcup_{x \in \bar{x}\left(T, \alpha_{k}\right)+\delta_{k}^{\prime} \mathbb{B}} \bigcup_{r \in[0,1]}\left((1-r) N(x, \alpha)+r \partial G_{\delta_{k}^{\prime}}(x, \alpha)\right)$;

(c') $r_{k}(t) \leq 0$ a.e. $t \in[S, T]$

(d') there exists an integrable function $K_{m}$ such that

$$
\left|q_{k}(t)\right|+\left|r_{k}(t)\right| \leq K_{m}(t) \quad \text { a.e. } \quad t \in[S, T] .
$$

It follows from $\left(a^{\prime}\right)$, Lemma 3.2 and Theorem 4.6 .2 of [18] that

$$
\left|\dot{p}_{k}(t)\right| \leq k_{f}(t)\left|p_{k}(t)\right|+v(t) \quad \text { and } \quad\left|\xi_{k}(t)\right| \leq N(t)
$$

where $N(t)=k_{f}(t)\left|p_{k}(t)\right|+v(t)$ and $v(t)=\left(\left|q_{k}(t)\right|+\left|r_{k}(t)\right|\right) L_{b, l}(t)$. By Gronwall's inequality, we have

$$
\left|p_{k}(t)\right| \leq L\left(\left|p_{k}(T)\right|+\|v\|_{1}\right), \quad \text { where } \quad L=\exp \left(\left\|k_{f}\right\|_{1}\right) .
$$

This implies, by making use of ( $\left.\mathrm{b}^{\prime}\right)$, that $\left\|p_{k}\right\|_{\infty} \leq M$, for some $M>0$. It follows from $\left(e^{\prime}\right)$ that

$$
\left|q_{k}(t)\right| \leq K_{m}(t) \quad \text { and } \quad\left|r_{k}(t)\right| \leq K_{m}(t) .
$$

We introduce the following contractions $\tilde{q}_{k}=\left(1+K_{m}(t)\right)^{-1} q_{k}$ and $\tilde{r}_{k}=\left(1+K_{m}(t)\right)^{-1} r_{k}$, in the directions of $q_{k}$ and $r_{k}$ respectively. Note that $\left\{\left\|\tilde{q}_{k}\right\|_{\infty}\right\}$ and $\left\{\left\|\tilde{r}_{k}\right\|_{\infty}\right\}$ are uniformly bounded by 1 .

Note also that $\left\{t \mapsto \int_{S}^{t} \xi_{k} \mathrm{~d} s\right\}$ is equicontinuous and uniformly bounded. By Arzela-Ascoli's Theorem and the Dunfort Pettis Criterion ([18], Thm. 2.5.1), we can ensure, by the extraction of a sequence, that $p_{k} \rightarrow p$ uniformly, with $p \in W^{1,1}, \int_{S}^{t} \xi_{k} \mathrm{~d} s \rightarrow \int_{S}^{t} \xi \mathrm{d} s$ uniformly to $\xi \in L^{1}, \dot{p}_{k} \rightarrow \dot{p}$ and $\xi_{k} \rightarrow \xi$ weakly in $L^{1}, \tilde{q}_{k} \rightarrow \tilde{q}$ and $\tilde{r}_{k} \rightarrow \tilde{r}$ weakly* in $L^{\infty}$, satisfying

(11) $(-\dot{p}(t), 0, \xi(t)) \in \operatorname{co} \partial_{x, u, v} H\left(t, \bar{x}\left(t, \alpha_{0}\right), p(t), q(t), r(t), \bar{u}(t), \bar{v}(t), \alpha_{0}\right)$, a.e. $t \in[S, T]$;

(12) $-p(T) \in \bigcup_{s \in[0,1]}\left((1-s) N\left(\bar{x}\left(T, \alpha_{0}\right), \alpha_{0}\right)+s \partial G_{0}\left(\bar{x}\left(T, \alpha_{0}\right), \alpha_{0}\right)\right)$;

(13) $r(t) \leq 0$ a.e. $t \in[S, T]$;

(14) there exists an integrable function $K_{m}$ such that

$$
|q(t)|+|r(t)| \leq K_{m}(t) \quad \text { a.e. } \quad t \in[S, T] .
$$


So, there exists $(p, q, r, \xi) \in \mathcal{M}\left(\alpha_{0}\right)$ satisfying

$$
\begin{aligned}
& a_{j}=\int_{S}^{T} r(t) \cdot l\left(t, \bar{x}\left(t, \alpha_{0}\right), \bar{u}(t), \bar{v}(t), \alpha_{0}\right) \mathrm{d} t \\
& b_{j}=\int_{S}^{T} \xi(t) \mathrm{d} t \quad \text { and } \\
& c_{j}=\int_{S}^{T}\left(|q(t)|+|r(t)|-k_{m}(t)|p(t)|\right) \mathrm{d} t
\end{aligned}
$$

where $q(t)=\left(1+K_{m}(t)\right) \tilde{q}(t)$ and $r(t)=\left(1+K_{m}(t)\right) \tilde{r}(t)$. Therefore, $x_{0} \in \mathfrak{T}\left(\alpha_{0}\right)$, i.e., $\left(\alpha_{0}, x_{0}\right) \in \operatorname{Gr} \mathfrak{T}$, what completes the proof of Lemma 5.2.

We now return to the proof of Theorem 4.4. By Lemma 5.2, $\mathfrak{T}(\alpha)$ is convex for each $\alpha \in \mathcal{A}$ and $\lim \sup \operatorname{Gr} \mathfrak{T}_{i} \subset$ $\operatorname{Gr} \mathfrak{T}$. Moreover, from $a^{\prime}$ ) and $c^{\prime}$ we deduce existence of

$$
\left(p_{i}(\cdot, \alpha), q_{i}(\cdot, \alpha), r_{i}(\cdot, \alpha), \xi_{i}(\cdot, \alpha)\right) \in \mathcal{O}_{\delta_{i}^{\prime}}(\alpha)
$$

Then, $\Gamma_{M}^{i}(\alpha) \in \mathfrak{T}_{i}(\alpha)$. By Lemma 2.1 there exists $\Gamma_{M}(\alpha) \in \mathfrak{T}(\alpha)$. Thus, by definition of the set $\mathfrak{T}(\alpha)$, there exists

$$
\left(p_{M}(\cdot, \alpha), q_{M}(\cdot, \alpha), r_{M}(\cdot, \alpha), \xi_{M}(\cdot, \alpha)\right) \in \mathcal{M}(\alpha)
$$

and we deduce that

$$
\int_{\mathcal{A}} r_{M}(t, \alpha) l(t, \bar{x}(t, \alpha), \bar{u}(t), \bar{v}(t), \alpha) \mathrm{d} \Lambda(\alpha)=0 \quad \text { a.e. } \quad t \in[S, T]
$$

and

$$
\int_{\mathcal{A}} \xi_{M}(t, \alpha) \mathrm{d} \Lambda(\alpha) \in \operatorname{co} N_{V(t)}(\bar{v}(t)) \quad \text { a.e. } \quad t \in[S, T]
$$

Furthermore, for some integrable function $k_{m}$,

$$
\int_{\mathcal{A}}\left(\left|q_{M}(t, \alpha)\right|+\left|r_{M}(t, \alpha)\right|-k_{m}(t)\left|p_{M}(t, \alpha)\right|\right) \mathrm{d} \Lambda(\alpha) \leq 0 \quad \text { a.e. } \quad t \in[S, T] .
$$

For each $M \in \mathbb{N}$, consider $\alpha \mapsto\left(p_{M}(\cdot, \alpha), q_{M}(\cdot, \alpha), r_{M}(\cdot, \alpha), \xi_{M}(\cdot, \alpha)\right)$ in the Hilbert space

$$
\mathcal{H}:=L^{2}\left(\mathcal{A} ; L^{2}\left([S, T] ; \mathbb{R}^{n} \times \mathbb{R}^{m_{b}} \times \mathbb{R}^{m_{l}} \times \mathbb{R}^{k_{v}}\right)\right),
$$

with inner product

$$
\begin{array}{r}
\left((p, q, r, \xi),\left(p^{\prime}, q^{\prime}, r^{\prime}, \xi^{\prime}\right)\right)_{\Lambda}=\int_{\mathcal{A}} \int_{S}^{T}\left[p(t, \alpha) p^{\prime}(t, \alpha)+q(t, \alpha) q^{\prime}(t, \alpha)+r(t, \alpha) r^{\prime}(t, \alpha)\right. \\
\left.+\xi(t, \alpha) \xi^{\prime}(t, \alpha)\right] \mathrm{d} t \Lambda(\mathrm{d} \alpha) .
\end{array}
$$


Then, the sequence $\left\{h_{M}\right\}_{M=1}^{\infty}$ given by $h_{M}(\alpha)=\left(p_{M}(\cdot, \alpha), q_{M}(\cdot, \alpha), r_{M}(\cdot, \alpha), \xi_{M}(\cdot, \alpha)\right)$, is uniformly norm bounded, i.e.,

$$
\left\|h_{M}\right\|=\int_{\mathcal{A}} \int_{S}^{T}\left|h_{M}(\alpha)(t)\right|^{2} \leq \text { const }, \quad \forall M
$$

and therefore a subsequence has a weak limit

$$
h(\alpha)=(p(\cdot, \alpha), q(\cdot, \alpha), r(\cdot, \alpha), \xi(\cdot, \alpha))
$$

But

$$
\mathcal{L}=\{h \in \mathcal{H} \mid h(\alpha) \in \mathcal{M}(\alpha), \Lambda \text { - a.e. } \alpha \in \mathcal{A}\}
$$

is a convex strongly closed subset of $\mathcal{H}$ and so $\mathcal{L}$ is weakly closed implying that

$$
(p(\cdot, \alpha), q(\cdot, \alpha), r(\cdot, \alpha), \xi(\cdot, \alpha)) \in \mathcal{M}(\alpha), \quad \Lambda-\text { a.e. } \quad \alpha \in \mathcal{A} .
$$

Therefore, due to the weak convergence of $\left\{h_{M}\right\}_{M=1}^{\infty}$, we deduce from (5.4)-(5.6) that

$$
\begin{gathered}
\int_{\mathcal{A}} r(t, \alpha) l(t, \bar{x}(t, \alpha), \bar{u}(t), \bar{v}(t), \alpha) \mathrm{d} \Lambda(\alpha)=0, \\
\int_{\mathcal{A}} \xi(t, \alpha) \mathrm{d} \Lambda(\alpha) \in \operatorname{co} N_{V(t)}(\bar{v}(t)) \quad \text { a.e. } \quad t \in[S, T]
\end{gathered}
$$

and

$$
\int_{\mathcal{A}}\left(|q(t, \alpha)|+|r(t, \alpha)|-k_{m}(t)|p(t, \alpha)|\right) \mathrm{d} \Lambda(\alpha) \leq 0 \quad \text { a.e. } \quad t \in[S, T]
$$

concluding the proof of Theorem 4.4.

\section{Conclusion}

We derived necessary conditions for weak local minimizers of minimax optimal control problems with equality and inequality constraints (Thm. 4.4), introducing appropiated constraint qualifications, namely full rank or Mangasarian-Fromovitz types for the mixed constraints. We showed through an example that the statement of Proposition 4.2, which provides necessary conditions for the case in which the set $\mathcal{A}$ of parameters is finite, is no longer valid when the set of parameters is infinite. We also presented an example to show that the Mangasarian-Fromovitz hypotheses are less restrictive than the full rank one, extending results in classical control theory.

As regards future work, it would be interesting to come up with new type of regularity conditions for minimax control problems. A recent publication [1] provided constant rank regularity conditions for mixed constrained optimal control problems and can serve as inspiration for minimax control problems.

As mentioned earlier in the introduction another theme for future exploration are the connections between the necessary optimality conditions for minimax control problems as developed in this work and the necessary optimality conditions obtained by [22] for bi-level control problems, since minimax control problems can be reformulated into bi-level models. 
Acknowledgements. The authors gratefully acknowledge the financial support from Coordenação de Aperfeicoamento de Pessoal de Nivel Superior - Brasil (CAPES) - Finance Code 001 and Sao Paulo Research Foundation - Grant numbers 2018/08036-8 and 2013/07375-0, the second grant received through the Center for Applied Mathematical Sciences Applied to Industry - CEPID-CeMEAI. The support of the Portuguese research projects POCI-01-0145-FEDER-028247-ToChair, POCI-01-0145-FEDER-031447-Upwind and NORTE-01-0145-FEDER-031411, Harmony, funded by FCT-Fundação para a Ciencia e Tecnologia through the Program P2020-COMPETE, is also gratefully acknowledged.

\section{REFERENCES}

[1] R. Andreani, V. Antunes de Oliveira, J. Tomaz Pereira and G. Nunes Silva, A weak maximum principle for optimal control problems with mixed constraints under a constant rank condition. IMA J. Math. Control Inf. 37 (2020) 1021-1047.

[2] M.R. de Pinho, Mixed constrained control problems. J. Math. Anal. Appl. 278 (2003) 293-307.

[3] M.R. de Pinho and A. Ilchmann, Weak maximum principle for optimal control problems with mixed constrained. Nonlinear Anal. 48 (2002) 1179-1196.

[4] M.R. de Pinho and J.F. Rosenblueth, Necessary conditions for constrained problems under Mangasarian-Fromowitz conditions. SIAM J. Control Optim. 47 (2008) 535-552.

[5] M.R. de Pinho and R.B. Vinter, Necessary conditions for optimal control problems involving nonlinear differential algebraic equations. J. Math. Anal. Appl. 212 (1997) 493-516.

[6] M.R. de Pinho, P. Loewen and G.N. Silva, A weak maximum principle for optimal control problems with nonsmooth mixed constraints. Set Valued Variat. Anal. 17 (2009) 203-221.

[7] M.R. de Pinho; R.B. Vinter and H. Zheng, A maximum principle for optimal control problems with mixed constraints. IMA J. Math. Control Inf. 18 (2001) 189-205.

[8] G.B. Folland, Real Analysis-Modern Techniques and Their Applications. John Wiley and Sons (1999).

[9] M.R. Hestenes, Calculus of Variations and Optimal Control Theory. Willey, New York (1966).

[10] D. Karamzin, V. de Oliveira, F. Pereira and G. Silva, Minimax optimal control problem with state constraints. Eur. J. Control 32 (2016) 24-31.

[11] A. Li and J.J. Ye, Necessary optimality conditions for optimal control problems with nonsmooth mixed state and control constraints. Set-Valued Var. Anal. 24 (2016) 449-470.

[12] A. Li and J.J. Ye, Necessary optimality conditions for implicit control systems with applications to control of differential algebraic equations. Set-Valued Var. Anal. 26 (2018) 179-203.

[13] B.S. Mordukhovich, Variational Analysis and Applications. Springer (2018).

[14] L.W. Neustadt, Optimization, A Theory of Necessary Conditions. Princeton University Press, Princeton, New Jersey (1976).

[15] N.P. Osmolovskii, Second order conditions for a weak local minimum in an optimal control problem (necessity and sufficiency. Soviet Math. Dokl. 16 (1975) 1480-1484.

[16] Z. Pales and V. Zeidan, First and second order necessary conditions for control problems with constraints. Trans. Am. Math. Soc. 346 (1994) 421-453.

[17] G. Stefany and P.L. Zezza, Optimality conditions for constrained control problems. SIAM J. Control Optim. 34 (1996) 635-659.

[18] R.B. Vinter, Optimal Control, Birkhauser Basel, Boston (2000).

[19] R.B. Vinter, Minimax optimal control. SIAM J. Control Optim. 44 (2005) 939-967.

[20] R.B. Vinter and G. Pappas, A maximum principle for nonsmooth-control problems with state constraints. J. Math. Anal. Appl. 89 (1982) 212-232.

[21] R.B. Vinter and H. Zheng, Necessary conditions for optimal control problems with state constraints. Trans. Am. Math. Soc. 350 (1998) 1181-1204.

[22] J.J. Ye, Necessary conditions for bilevel dynamic optimization problems. SIAM J. Control Optim. 33 (1995) $1208-1223$. 